\title{
Some numerical studies of interface advection properties of level set method
}

\author{
A SALIH ${ }^{1}$ and $\mathrm{S}$ GHOSH MOULIC ${ }^{2}$ \\ ${ }^{1}$ Department of Aerospace Engineering, Indian Institute of Space Science and \\ Technology, Thiruvananthapuram 695022 \\ ${ }^{2}$ Department of Mechanical Engineering, Indian Institute of Technology, \\ Kharagpur 721302 \\ e-mail: salih@iist.ac.in
}

MS received 18 December 2007; revised 31 July 2008

\begin{abstract}
In this paper, we discuss the results of a series of tests carried out to assess the level set methodology for capturing interfaces between two immiscible fluids. The tests are designed to investigate the accuracy of convection process, the preservation of interface shape, and the mass conservation properties of individual fluids. These test cases involve the advection of interfaces of different shapes exposed to translation, rotation, deformation, and shear flow. Prescribed solenoidal velocity fields are used and no attempt is made to couple the advection of the level set function with the momentum equations. For the solution of level set equation we have employed first-order upwind scheme, MacCormack method, second-order ENO scheme, and fifth-order WENO scheme. Our studies show that the level set method perform well when higher-order schemes are used for the solution of advection equation. However, for certain type of shearing and vortical velocity fields mass conservation is an issue on coarser meshes even with higher order schemes. Finer mesh must be used in such situations to reduce numerical diffusion.
\end{abstract}

Keywords. Level set method; interface advection; numerical diffusion; mass conservation.

\section{Introduction}

Over the years a number of computational methods have been developed for solving flow with moving fluid interfaces. There are many engineering applications where flows involving moving interfaces are encountered. A prominent area is the multi-phase flows. More often, in a multi-phase flow simulation the location of fluid interface is not known a priori. Hence any solution procedure for computing such flows must include a method for locating and advancing the interface. An accurate and robust numerical scheme is required for this purpose. The literature in the broad area of multi-phase flow simulation with moving interface is quite extensive and a comprehensive discussion is beyond the intent of this paper. However, below we give a brief account of some of the important methods for computing multi-phase flow involving moving interfaces with particular emphasis on level set method. 


\subsection{Methods of computing flow with interfaces}

Depending on the scheme used for locating the interface, the available algorithms can be categorized as interface fitting methods, interface tracking methods, and interface capturing methods. Interface fitting methods solve for the flow only within a single phase by treating the interface as a free surface and placing it at the boundary of the computational domain. The motion of the free surface is accounted for by a coordinate transformation which maps the moving body-fitted coordinate system in physical space to a fixed, uniformly spaced coordinate system in computational space (see for example, Kang \& Leal (1987)). A boundaryfitted grid arrangement maintains the sharpness of interface and helps to apply the interfacial conditions at the exact locations without any smearing or redistribution. However, when the interface becomes highly distorted, it is difficult to adequately resolve the geometrical complexities while maintaining the desired mesh control. Mesh skewness and stretching may impact additional complexities when interfaces merge or break-ups. The interface tracking and interface capturing methods avoid grid-related problems associated with interface fitting methods by employing a grid which is fixed in the physical domain and by defining the location of the interface relative to the fixed grid. The principal difference between the interface tracking and interface capturing schemes is the manner in which the location of the interface is identified.

Interface tracking method may be further classified as front tracking and volume tracking. Front tracking methods have been used extensively by the Glimm group (Chern et al 1986, Glimm et al 1987, Glimm et al 1998) and by the Tryggvason group (Unverdi \& Tryggvason 1992, Juric \& Tryggvason 1998, Tryggvason \& Unverdi 1990, Tryggvason et al 1998). Glimm and co-workers employed a front tracking method where a separate front marks the interface but a fixed grid, only modified near the front to make a grid line follow the interface, is used for the fluid within each phase. Tryggvason and co-workers have achieved remarkable success in two- and three-dimensional multi-phase flow simulations. Essentially front tracking involves explicit computational elements moving through an Eulerian grid. The advantage of front tracking method is their ability to resolve features of the interface that are smaller than the mesh spacing of the regular Eulerian grid on which the interface is overlaid. This feature of the front tracking methods helps to improve the accuracy of computation related to interface which is important in modelling surface tension (Popinet \& Zaleski 1999). The major disadvantage of the front tracking method is that the topological changes in the interface, like merging and break-ups requires intensive logical manipulation and awkward subjective methods must be used to add or remove marker points as they get too far apart or too close together.

Different versions of volume tracking methods can be found in the literature. Among them the most popular are the Marker-and-Cell (MAC) method of Harlow \& Welch (1965) and the widely used Volume-of-Fluid (VOF) of Nichols \& Hirt (1975). The MAC method is based on a fixed, Eulerian grid of control volumes in which massless marker particles are assumed to be distributed throughout the grids. Evolution of the free surface was computed by advecting the markers with locally interpolated fluid velocities. Some special treatments were required to define the fluid properties in the newly filled cells and to cancel values in cells that are emptied. One reason for the success of marker particle method is that it offers the distinct advantage of eliminating all logic problems associated with merging or fragmentation of surfaces. This is primarily a consequence of the fact that while particles have to be ordered with well defined neighbours when marking an interface they do not have to be well ordered when marking regions. The MAC method has been used primarily for two-dimensional simulations because it requires considerable memory and CPU time to accommodate the necessary number of 
marker particles. Another limitation of the method is that they donot do a very good job of following flow processes in regions involving converging/diverging flows. In recent years, a variety of improvements have contributed to an increase in the accuracy and applicability of the original MAC method, see for example, Tomé et al (2000) and Armenio (1997).

In contrast to the marker particle used in MAC method, VOF use the fraction of a cell volume occupied by one of the fluids as the marker quantity. A scalar function $F(x, y, t)$ is defined to represent the fraction of liquid contained in a cell. This fraction is zero for a cell which does not occupy fluid and there is no interface in that cell. Conversely, the function is unity for a cell which is completely occupied by the fluid and again no interface present in that cell. An interface is to be constructed only when $F$ is a fraction between 0 and 1 . The function $F$ was updated using a transport equation

$$
\frac{\partial F}{\partial t}+(\mathbf{u} \cdot \nabla) F=0
$$

to model the convection of $F$. As with the MAC method, only the flow field within the liquid and free surface cells were computed. For the purpose of computations, the interface is approximated by a straight line through the cell. The location and slope of the line are determined by the average value of $F$ in the cell and by the gradient of $F$. Since there is only one piece of information regarding the interface per cell available, a certain arbitrariness in reconstructing the shape of the interface has to be allowed. More accurate VOF techniques attempt to fit the interface through piecewise linear interface construction (PLIC) methods. A significant improvement in this regard was made by Youngs with the PLIC algorithm (Youngs 1982). Since then, some improvements in the reconstruction of the VOF interface have been reported (Ashgriz \& Poo 1991, Rider \& Kothe 1998). The modelling of surface tension in the context of VOF methods is proposed by Brackbill et al (1992). More recently an interesting idea of a second-order VOF tracking method, employing an approximate projection operator, has been proposed by Puckett et al 1997. The VOF methods for capturing flows with interfaces have thoroughly reviewed by Scardovelli \& Zaleski (1999). A comparison of various VOF methods can be found in Rudman (1997).

The interface capturing methods are a class of method where a separate phase function, discretized on the fixed Eulerian grid, is used to indicate which phase is present at a given location. The interface is implicitly defined (captured) as the location of the discontinuity in the phase function. This idea is similar in spirit to shock capturing approach which has been used extensively for the simulation of compressible flows with shock waves. The interface motion is simply obtained by advection of the corresponding phase function. The advection equation for the phase function is usually solved by the technology borrowed from hyperbolic conservation laws. The primary advantage of the interface capturing methods is that it eliminate the need for special tracking procedures associated with interface tracking methods and handle large distortions and topology changes of interfaces in a more natural way.

\subsection{Level set method}

Among several kinds of interface capturing methods the level set method introduced by Osher \& Sethian (1988) represents a classic interface capturing scheme. Level set method offer highly robust and accurate methods for capturing interfaces moving under complex motions. The key idea was the Hamilton-Jacobi approach to numerical solutions of time dependent equation for a moving implicit surface. The basic idea is as follows. The interface is modelled as the zero level set of a smooth phase function (level set function), $\phi$, defined over the whole 
computational domain. This level set function is advected with the background flow field and thus propagating the interface exactly as the zero level set of $\phi$. Since the interface is captured implicitly, this algorithm is capable of capturing the intrinsic geometric properties of highly complicated interfaces in a quite natural way. Further, it automatically takes care of topological changes in the interface such as merging and breaking ups.

Since its introduction, the level set methods have seen tremendously expanded applications in such diverse areas like, two-phase flows, combustion, crystal growth, image processing, computer vision, etching, and grid generation to name a few. The early applications of fluid dynamics problems using level set methods to capture the interface can be found in the paper by Mulder et al (1992) and Zhu \& Sethian (1992). The first paper discusses the motion of interface in an inviscid, compressible flows and the second is about the combustion and propagation of cold flame from burnt region to unburnt region. These two works were followed by the level set method applied to the motion of interfaces in incompressible flows. Sussman et al (1994) presented results for several two-dimensional cases involving the motion of air bubbles in water and falling water drops in air. Later, Chang et al (1996) gave the detailed derivation of level set based governing equations of motion for incompressible flows.

An hybrid method combining the level set method with immersed interface method of LeVeque \& Li (1994) was developed by Hou et al (1997). They applied this method for studying Hele-Shaw flow, an unstable flow involving two fluids with very different viscosity. An adaptive level set method was developed by Sussman et al (1999) for solving incompressible two-phase flow with surface tension. Fedkiw et al (1999) introduced level set based ghost fluid method in which two-phase contact discontinuity is discretized with a technique similar to those used for the solid-wall boundary. The basic idea is to extrapolate the real solution values in each phase onto fictitious ghost nodes located in the other phase, and then solve the governing equations in both phases separately. The important feature of this method lies in the fact that the property discontinuities and jump conditions at the interface are well preserved. Sussman \& Puckett (2000) combined the VOF and level set methods in order to alleviate some of the geometrical problems of the VOF method. The resulting scheme is completely Eulerian and does not incorporate any of the front tracking characteristics in capturing the interface. This hybrid method improves the mass conservation in level set method in underresolved regions. Recently, Enright et al (2002) used Lagrangian marker particles to rebuild the level set in regions which are under-resolved. Their hybrid particle level set method is capable of maintaining very sharp interfaces. More information on level set methods can also be found in the recent review articles by Osher \& Fedikiw (2001) and Sethian \& Smereka (2003) as well as the recent books of Sethian (1999) and Osher \& Fedikiw (2003).

A particular advantage of level set method over the popular VOF method is in calculating the geometrical properties of the interface. In VOF method calculation of local unit normal and curvature of the interface requires special procedures and is not straight forward. On the other hand, level set method offers very straight forward and elegant way to compute the local normal and curvature of the interface. However, it is a well known fact that like any interface capturing scheme, the level set method suffers from numerical diffusion in the vicinity of the interface. This may cause the sharp edges of the interface to be smoothed out and consequently the volume of individual fluid (in the case of incompressible flows) may not be conserved as the interface evolves. A survey of the literature will reveal that a systematic study to assess the level set methodology for capturing interfaces between two immiscible fluids is absent. Whereas, the volume-of-fluid method and various front tracking schemes are routinely tested and validated using several standard test problems (see for example, Ashgriz \& Poo (1991), Lafaurie et al (1994), Rudman (1997), Rider \& Kothe (1995), Tryggvason et al (1998)). Thus, 
in the present paper, we attempt a series of rigorous tests to assess the performance of level set method for capturing interfaces between two immiscible fluids. The tests are designed to investigate the accuracy of convection process, the preservation of interface shape, and the volume conservation properties of individual fluids.

\section{Level set formulation and discretization}

In the level set method, the interface between the two fluid phases is represented by a continuous scalar function $\phi(\mathbf{x}, t)$ defined over the complete computational domain and is set equal to zero on the interface. The level set function at any given point is taken as the signed normal distance from the interface with positive on one side, and negative on the other. This way both phases are identified, and the location of the fluid interface is associated with the surface $\phi=0$. The motion of the interface is determined by the underlying velocity field $\mathbf{u}$, which may depend on a variety of factors including position, time, and geometry of the interface. In multi-phase flow simulation the velocity field normally is externally given and the evolution of the interface can be determined by solving the following transport equation

$$
\frac{\partial \phi}{\partial t}+\mathbf{u} \cdot \nabla \phi=0 \quad \text { for } t>0 \quad \text { with } \phi(\mathbf{x}, 0)=\phi_{0}(\mathbf{x})
$$

where $\phi_{0}$ embeds the initial location of the interface. The fluid interface being a material surface provided that there is no mass transfer across the interface, it is always identified with zero level set of $\phi(\mathbf{x}, t)$. It must be noted that, the level set equation 1 does not represents any physical conservation law and thus, there is no guarantee that the mass of the individual fluid phase would be conserved during the evolution of level set function. When the level set algorithm is coupled to the Navier-Stokes equation, numerical instability can arise because of the discontinuous nature of density and viscosity across the fluid interface. In order to avoid this, fluid properties have to be smoothed across the interface (Sussman et al 1994). Since the present study involves passive advection of level set function in a prescribed fluid without coupling to the Navier-Stokes equation the smoothing of fluid properties (if they are different across the interface) would not be required at all. As a consequence, we do not have to reinitialize the level set function which is otherwise required (Sussman et al 1994).

As we have mentioned earlier the level set transport equation is a hyperbolic equation and a rich class of algorithms are available for its solution, some naive and some sophisticated. In the present work we employ the first-order upwind scheme, MacCormack method, second-order ENO scheme, and fifth-order WENO scheme. For the sake of completeness, the discretization algorithms of the above mentioned methods are given in Appendix I. The temporal discretization for all the schemes except MacCormack is carried out by either Forward Euler time stepping or the second-order TVD RK method proposed by Shu \& Osher (1988). The use of explicit time marching scheme imposes a stability constraint on time step which may be enforced using the Courant-Friedrichs-Lewy (CFL) condition given by

$$
C F L=\max _{i, j}\left\{\frac{\left|u_{i, j}^{n}\right|}{\Delta x}+\frac{\left|v_{i, j}^{n}\right|}{\Delta y}\right\} \Delta t,
$$

where $i, j$ are the grid indices and $n$ the time level. For stability, $0<C F L<1$. The performance of each of the schemes in advecting the level set function will be compared for different types of test problems. For the purpose of comparison, the solution error in $\phi$ is 
measured in terms of how much the computed interface differers from the expected interface as suggested by Sussman \& Fatemi (1999). This first-order accurate error measure is given by

$$
E_{\phi}=\frac{1}{L} \sum_{i, j}\left|H\left(\phi_{e}\right)-H\left(\phi_{c}\right)\right| \Delta x \Delta y \quad \text { for all }(i, j) \in \Omega,
$$

where $L$ is the perimeter of the expected interface, $\phi_{e}$ the level set function representing the expected interface, $\phi_{c}$ the level set function representing the computed interface, and $H$ is the smeared out heaviside function defined by equation

$$
H_{\varepsilon}(\phi)= \begin{cases}0, & \text { if } \phi<-\varepsilon \\ \frac{\phi+\varepsilon}{2 \varepsilon}+\frac{1}{2 \pi} \sin \left(\frac{\pi \phi}{\varepsilon}\right), & \text { if }|\phi| \leq \varepsilon \\ 1, & \text { if } \phi>\varepsilon\end{cases}
$$

with the parameter $\varepsilon$ taken as one-half of the grid size.

In addition to measuring the error in $\phi$, we will also estimate the volume error (area error in two-dimensional case) during the computation period for all test cases. The normalized fluid volume enclosed by an interface at any instant of time is defined as

$$
\mathcal{V}^{\prime}(t)=\frac{\mathcal{V}(t)}{\mathcal{V}_{0}}
$$

where $\mathcal{V}_{0}$ is the initial volume enclosing the interface and $\mathcal{V}(t)$ is the corresponding computed volume at time $t$. Also the percentage volume loss (area loss in two-dimensional case) at any instant of time can be estimated as $\delta \mathcal{V}(t)=\left(1-\mathcal{V}^{\prime}(t)\right) \times 100$. The volume of fluid enclosing the interface (where $\phi$ taken as positive) is calculated using integral relation

$$
\mathcal{V}(t)=\int_{\Omega} H_{\varepsilon}(\phi(\mathbf{x}, t)) d \mathscr{V},
$$

where the integration is carried over the entire domain $\Omega$, since $H(\phi(\mathbf{x})$ prunes out the subdomain outside the interface. The above integral can be numerically estimated as follows

$$
\sum_{j=1}^{n} \sum_{i=1}^{m}\left(H_{\varepsilon}(\phi)\right)_{i, j} \Delta x \Delta y
$$

where $m$ and $n$ are the number of control volumes in $x$ - and $y$-directions respectively.

\section{Test problems and results}

There are seven specific test cases that we have considered. Some simple and other very stringent. Simple problems like pure translation and rigid body rotation serve as useful debugging tests. More stringent test problems having flows that bring about topology change are useful to assess the strengths and weaknesses relevant to modelling interfacial flows. The seven test problems are, the advection of a circular interface in an oblique unidirectional velocity field, the advection of a square interface in the same velocity field, the rotation of a square interface in a purely rigid body rotational velocity field, the advection of a circular interface in a time-reversed shear velocity field, the advection of a circular interface in a single-vortex 
velocity field, the advection of a circular interface in a time-reversed single-vortex velocity field, and finally the advection of a circular interface in a time-reversed deformation velocity field. We remark here that the prescribed velocity field in all these test cases are solenoidal. Typically we perform the tests on four different mesh sizes of $40 \times 40,80 \times 80,160 \times 160$, and $320 \times 320$. The time step is chosen so as to satisfy the $C F L$ condition for advection. The details of the tests are described below.

\subsection{Translation of circular interface in uniform flow}

This simple test case consists of passively advecting a circular interface obliquely (to make the advection direction inclined to coordinate axes) across a two-dimensional domain $\Omega=$ $[0,1] \times[0,1]$. The radius of the circle initially placed at the center of the domain, is $0 \cdot 1$. The circle is translated in a uniform periodic velocity field prescribed by

$$
u=1, \quad v=-1 .
$$

After advecting for $t=0 \cdot 3$, the theoretical new center is at $(0 \cdot 8,0 \cdot 2)$. The $C F L$ number used is 0.4 . Figure 1 displays the results of computation performed on a $80 \times 80$ mesh along with prescribed velocity field and the initial and final location (exact) of the interface. Tables 1 and 2 respectively give the summary of solution errors and \% area loss for different schemes and for different mesh sizes. From the figure it is seen that barring upwind method,
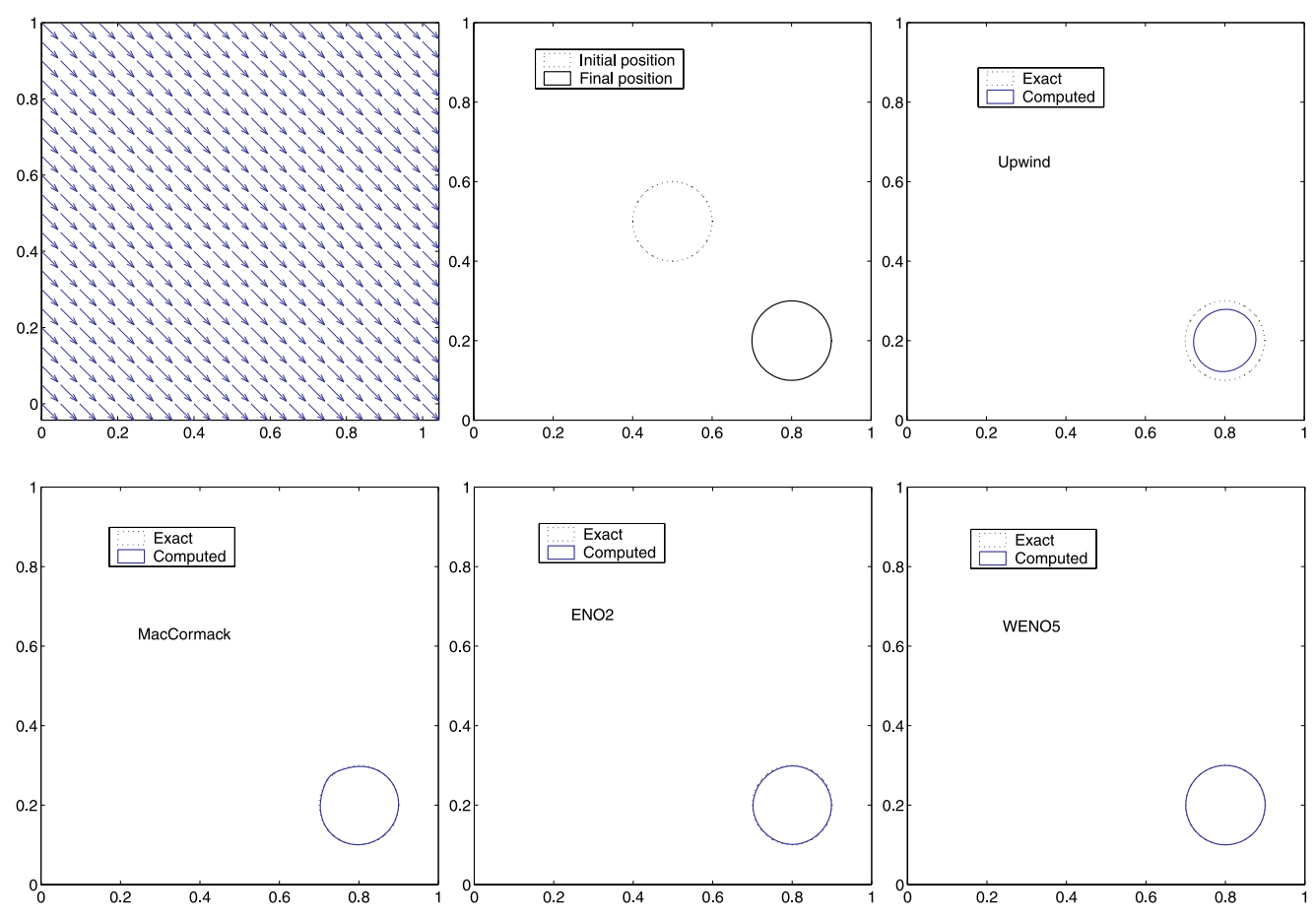

Figure 1. The prescribed uniform velocity field (top-left); initial and final location of the circle (top-center); remaining figures display the computed results on $80 \times 80$ grid by various schemes at $t=0 \cdot 3$. 
Table 1. Summary of error in $\phi$ for translation of circular interface using different schemes.

\begin{tabular}{ccccccccc}
\hline & \multicolumn{2}{c}{ Upwind } & \multicolumn{2}{c}{ MacCormack } & \multicolumn{2}{c}{ ENO-2 } & \multicolumn{2}{c}{ WENO-5 } \\
\cline { 2 - 9 } Grid & $E_{\phi}$ & order & $E_{\phi}$ & order & $E_{\phi}$ & order & $E_{\phi}$ & order \\
\hline $40^{2}$ & $4 \cdot 142-02$ & - & $4 \cdot 123-03$ & - & $7 \cdot 301-03$ & - & $3 \cdot 342-04$ & - \\
$80^{2}$ & $1 \cdot 920-02$ & $1 \cdot 109$ & $1 \cdot 201-03$ & $1 \cdot 779$ & $1 \cdot 691-03$ & $2 \cdot 110$ & $5 \cdot 662-05$ & $2 \cdot 561$ \\
$160^{2}$ & $8 \cdot 387-03$ & $1 \cdot 194$ & $1 \cdot 892-04$ & $2 \cdot 667$ & $4 \cdot 152-04$ & $2 \cdot 026$ & $1 \cdot 412-05$ & $2 \cdot 004$ \\
$320^{2}$ & $3 \cdot 926-03$ & $1 \cdot 095$ & $4 \cdot 362-05$ & $2 \cdot 116$ & $9 \cdot 503-05$ & $2 \cdot 127$ & $3 \cdot 536-06$ & 1.997 \\
\hline
\end{tabular}

all other schemes give excellent results. The computed profile and the exact profiles are almost indistinguishable. From the tables it can be seen that with all the schemes, the error in $\phi$ and the area loss reduces as the mesh is being refined. WENO-5 gives the most accurate results among all the advection schemes. On the finest grid, it gives an error in $\phi$ as low as $3.536 \times 10^{-6}$ and the area loss is $0.00005 \%$. Though this simple problem does not rigourously test the capability of the level set method we use this test to validate the advection schemes.

\subsection{Translation of square interface in uniform flow}

Square interface tests were reported by Lafaurie et al (1994), Rudman (1997), and Deborah (2004) in the context of VOF methods. This test case is essentially the same as that of the one described above except that the circular interface is now being replaced by a square interface having sides $0 \cdot 2$. All other test details remain the same. The advection of square interface involves more challenge in the sense the square interface have sharp corners as opposed the smooth circular interface. This problem is a good indicator of diffusion and dispersive errors and thus forms a good test for assessing how accurately the level set interface capturing scheme preserve the sharp interface shapes while advecting in a uniform flow. Thus, with an accurate scheme, the square interface should be translated intact towards the bottom right-hand corner of the domain. In figure 2 we display the results of computation performed on a $80 \times 80$ grid along with prescribed velocity field and the initial and final location (exact) of the interface. We observe that the interface translated using upwind scheme is clearly distorted, the square interface looks more like a circle. This smoothing out of the sharp corners of interface can be attributed to the large numerical diffusion present in the first order upwind scheme. This is

Table 2. Summary of $\%$ area loss for translation of circular interface using different schemes. Exact area enclosing the interface $=3 \cdot 1416-02$.

\begin{tabular}{rrrrrrrrr}
\hline & \multicolumn{2}{c}{ Upwind } & \multicolumn{2}{c}{ MacCormack } & \multicolumn{2}{c}{ ENO-2 } & \multicolumn{2}{c}{ WENO-5 } \\
\cline { 2 - 9 } Grid & Area & $\%$ loss & Area & $\%$ loss & Area & $\%$ loss & Area & $\%$ loss \\
\hline \multirow{2}{*}{$40^{2}$} & $5 \cdot 86-03$ & $81 \cdot 34$ & $3 \cdot 24-02$ & $-3 \cdot 21$ & $2 \cdot 73-02$ & $12 \cdot 95$ & $3 \cdot 12-02$ & $0 \cdot 57$ \\
$80^{2}$ & $1 \cdot 94-02$ & $38 \cdot 22$ & $3 \cdot 10-2$ & $1 \cdot 25$ & $3 \cdot 03-02$ & $3 \cdot 36$ & $3 \cdot 14-02$ & $0 \cdot 022$ \\
$160^{2}$ & $2 \cdot 62-02$ & $16 \cdot 73$ & $3 \cdot 14-02$ & $0 \cdot 0531$ & $3 \cdot 12-02$ & $0 \cdot 78$ & $3 \cdot 14-02$ & $0 \cdot 001$ \\
$320^{2}$ & $2 \cdot 89-02$ & $7 \cdot 84$ & $3 \cdot 14-02$ & $0 \cdot 002$ & $3 \cdot 13-02$ & $0 \cdot 18$ & $3 \cdot 14-02$ & $0 \cdot 00005$ \\
\hline
\end{tabular}



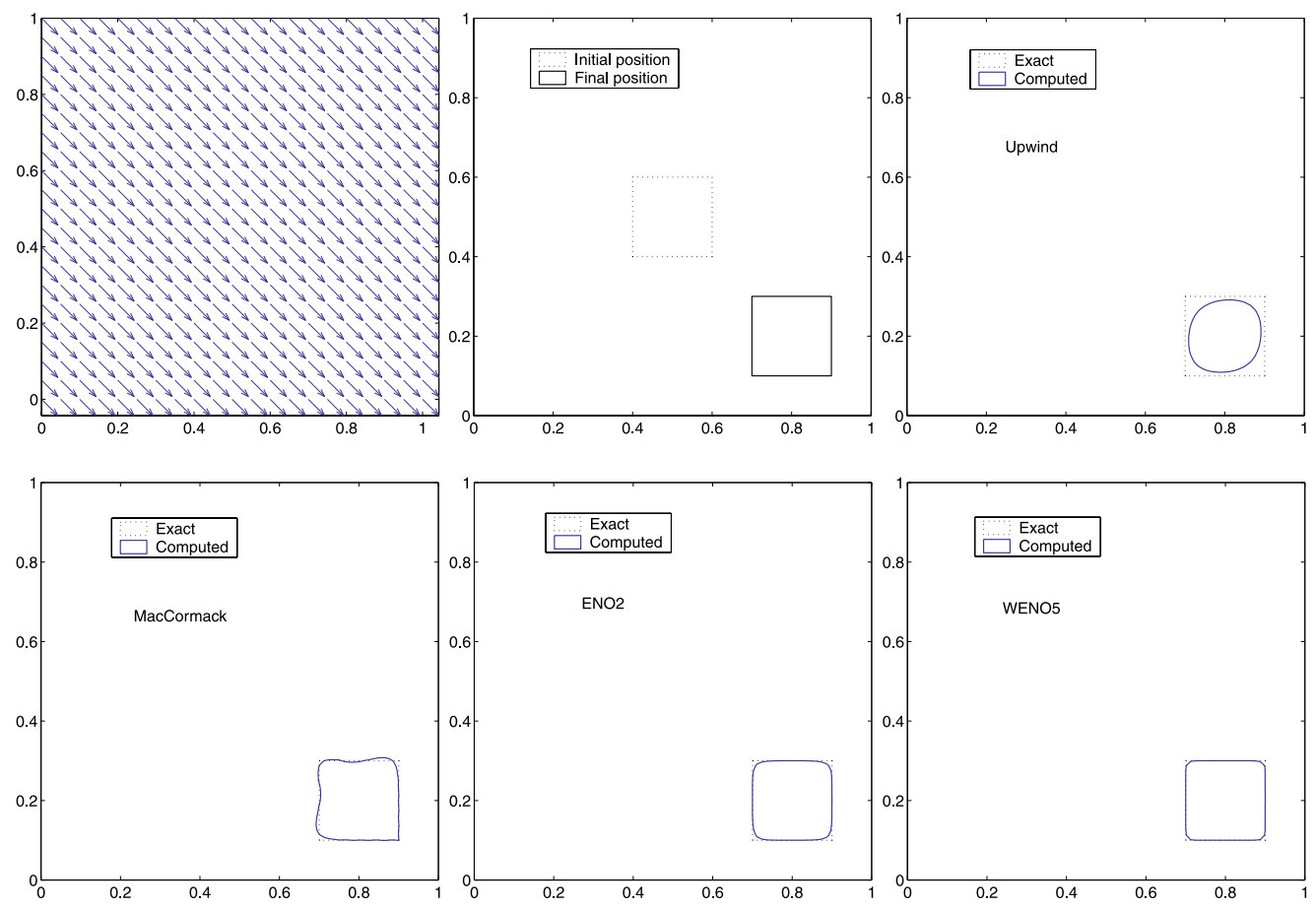

Figure 2. The prescribed uniform velocity field (top-left); initial and final location of the square (top-center); remaining figures display the computed results on $80 \times 80$ grid by various schemes at $t=0 \cdot 3$.

typical of upwinding type schemes. The interface translation using MacCormack's method develops waviness along the sides of the square though it conserves the total area very well. This behaviour of MacCormack's scheme is not unexpected because the scheme is known to introduce dispersive errors. Second order ENO scheme is slightly diffusive as can be seen from the slight rounding-off of the sharp corners. The result of the WENO scheme is the most accurate one in terms of preserving the integrity of the interface. Tables 3 and 4 respectively give the summary of solution errors and $\%$ area loss for different schemes on different mesh sizes.

Table 3. Summary of error in $\phi$ for translation of square interface using different schemes.

\begin{tabular}{rcccccccc}
\hline & \multicolumn{2}{c}{ Upwind } & \multicolumn{2}{c}{ MacCormack } & \multicolumn{2}{c}{ ENO-2 } & \multicolumn{2}{c}{ WENO-5 } \\
\cline { 2 - 9 } Grid & $E_{\phi}$ & order & $E_{\phi}$ & order & $E_{\phi}$ & order & $E_{\phi}$ & order \\
\hline $40^{2}$ & $3 \cdot 436-02$ & - & $5 \cdot 098-03$ & - & $5 \cdot 513-03$ & - & $2 \cdot 835-03$ & - \\
$80^{2}$ & $1.574-02$ & $1 \cdot 126$ & $2 \cdot 417-03$ & $1 \cdot 076$ & $2 \cdot 297-03$ & 1.263 & $8 \cdot 639-04$ & 1.714 \\
$160^{2}$ & $7 \cdot 589-03$ & 1.052 & $1 \cdot 172-03$ & 1.044 & $9 \cdot 372-04$ & 1.293 & $3.493-04$ & 1.306 \\
$320^{2}$ & $3 \cdot 811-03$ & 0.993 & $5 \cdot 346-04$ & $1 \cdot 132$ & $4 \cdot 008-04$ & $1 \cdot 225$ & $1.425-04$ & 1.294 \\
\hline
\end{tabular}


Table 4. Summary of $\%$ area loss for translation of square interface using different schemes. Exact area enclosing the interface $=4 \cdot 0-02$.

\begin{tabular}{|c|c|c|c|c|c|c|c|c|}
\hline \multirow[b]{2}{*}{ Grid } & \multicolumn{2}{|c|}{ Upwind } & \multicolumn{2}{|c|}{ MacCormack } & \multicolumn{2}{|c|}{ ENO-2 } & \multicolumn{2}{|c|}{ WENO-5 } \\
\hline & Area & $\%$ loss & Area & $\%$ loss & Area & $\%$ loss & Area & $\%$ loss \\
\hline $40^{2}$ & $1 \cdot 31-02$ & $67 \cdot 19$ & $3 \cdot 96-02$ & 0.96 & $3 \cdot 59-02$ & $10 \cdot 22$ & 3.79-02 & $5 \cdot 21$ \\
\hline $80^{2}$ & $2 \cdot 75-02$ & $31 \cdot 30$ & 4.04-02 & -0.95 & $3 \cdot 82-02$ & $4 \cdot 52$ & $3 \cdot 93-02$ & 1.64 \\
\hline $160^{2}$ & $3 \cdot 40-02$ & 15.04 & $4.00-02$ & 0.015 & $3.93-02$ & 1.78 & $3.97-02$ & 0.76 \\
\hline $320^{2}$ & $3 \cdot 70-02$ & 7.49 & $4.00-02$ & 0.0025 & $3.97-02$ & 0.69 & $3 \cdot 99-02$ & $0 \cdot 28$ \\
\hline
\end{tabular}

\subsection{Rotation of square interface}

Our next test case involves the rigid body rotation of the same square interface described above. The square interface is centered at the mid point of the computational domain $\Omega=$ $[0,1] \times[0,1]$ with its side initially parallel to coordinate axes. This square interface is made to undergo rigid body rotation about the center of the square in a constant vorticity velocity field given by

$$
u=y-1 / 2, \quad v=1 / 2-x
$$
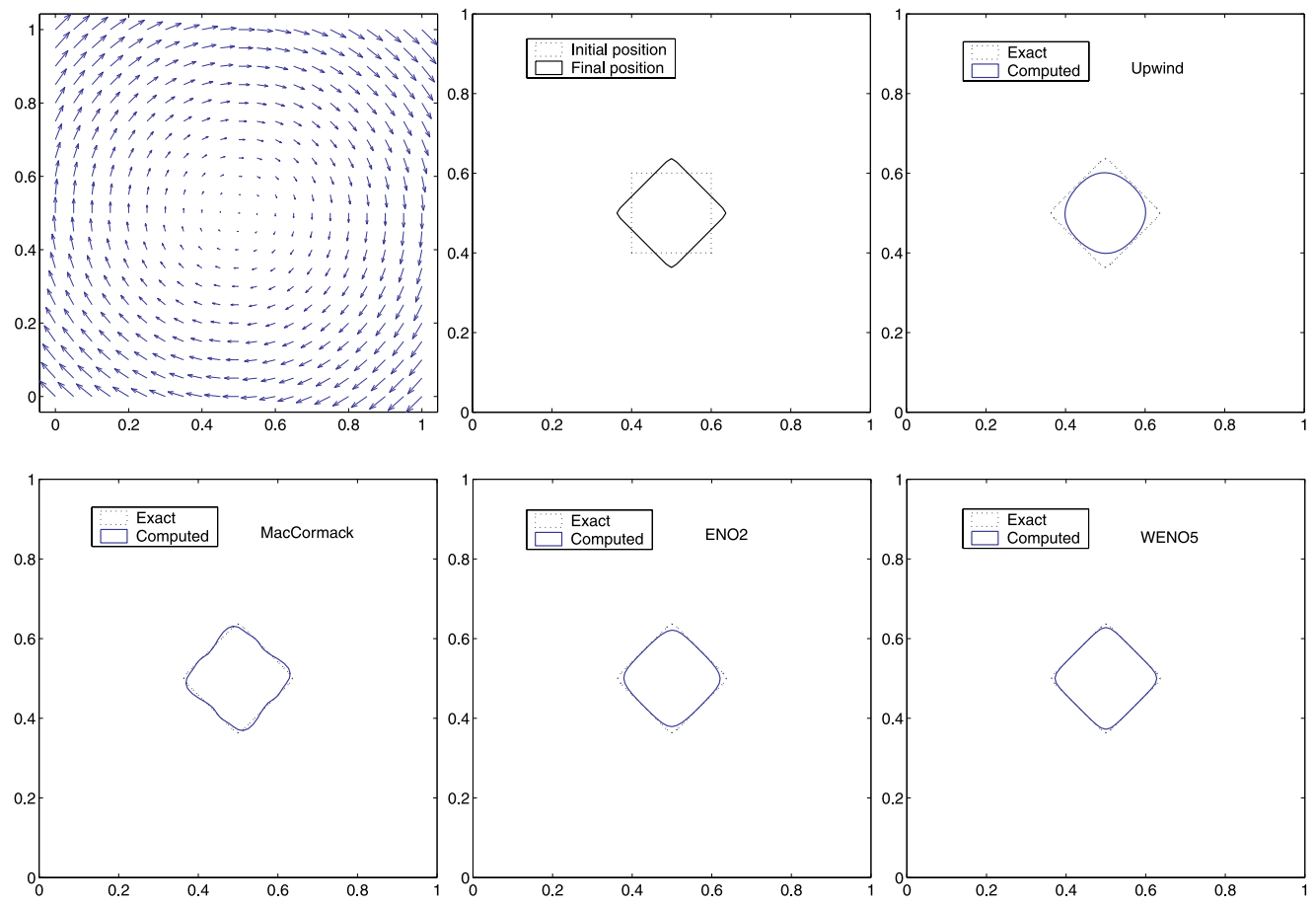

Figure 3. The prescribed rotational velocity field (top-left); initial and final location after $135^{\circ}$ rotation (top-center); remaining figures display the computed results on $80 \times 80$ grid by various schemes at $t=2 \cdot 3562$, which corresponds to $135^{\circ}$ rotation. 
Table 5. Summary of error in $\phi$ for rotation of square interface using different schemes.

\begin{tabular}{rcccccccc}
\hline & \multicolumn{2}{c}{ Upwind } & \multicolumn{2}{c}{ MacCormack } & \multicolumn{2}{c}{ ENO-2 } & \multicolumn{2}{c}{ WENO-5 } \\
\cline { 2 - 9 } Grid & $E_{\phi}$ & order & $E_{\phi}$ & order & $E_{\phi}$ & order & $E_{\phi}$ & order \\
\hline $40^{2}$ & $2 \cdot 156-02$ & - & $9 \cdot 263-03$ & - & $9 \cdot 289-03$ & - & $4.779-03$ & - \\
$80^{2}$ & $1 \cdot 153-02$ & 0.903 & $3.695-03$ & 1.326 & $3.532-03$ & 1.395 & $1 \cdot 348-03$ & 1.826 \\
$160^{2}$ & $6 \cdot 309-03$ & $0 \cdot 870$ & $1.610-03$ & 1.198 & $1.431-03$ & 1.303 & $3.520-04$ & 1.937 \\
$320^{2}$ & $3 \cdot 331-03$ & 0.921 & $5.955-04$ & 1.435 & $4.985-04$ & 1.521 & $1.180-04$ & 1.577 \\
\hline
\end{tabular}

for a time period $t=3 \pi / 4$ which corresponds to $135^{\circ}$ rotation (see figure 3 ). As in the previous cases, the $C F L$ number is taken as 0.4 . This is another simple but useful test as it involves advection of sharp interface in a rotational flow field. In figure 3 we display the results of computation performed on a $80 \times 80$ mesh along with prescribed constant vorticity velocity field and the initial and final location (exact) of the interface. The exact solution (dotted line) is also plotted on figures displaying computed profile for the sake of comparison. The behaviour of various schemes for this test is essentially similar to the case of translation of the rectangle. Upwind and MacCormack schemes do not preserve the interface shapes. Upwind produces a smeared out profile while MacCormack distorts the straight sides of the square interface. In this test also we see that the WENO scheme out performs other schemes. Tables 5 and 6 respectively give the summary of solution errors and \% area loss for different schemes on different mesh sizes.

\subsection{Circular interface in time-reversed shear field}

In the previous test cases we have considered, the interface is not supposed to undergo any deformation of shape during the advection in the prescribed velocity fields. Even with such restrictive constraints on the velocity field, it is seen that good translation and rotation of a sharp interface on an Eulerian mesh is still a difficult task. In real interfacial flows the situation is far more complicated than merely preserving the initial shape, as the interface deforms considerably. Thus it is important to assess the performance of interface capturing schemes in the presence of fluid shear. Such a velocity field is considered by Rudman (1997), Ubbink \& Issa (1999), and Deborah (2004) in the context of VOF methods, and is given by

Table 6. Summary of $\%$ area loss for rotation of square interface using different schemes. Exact area enclosing the interface $=4 \cdot 0-02$.

\begin{tabular}{rrrrrrrrr}
\hline & \multicolumn{2}{c}{ Upwind } & \multicolumn{2}{c}{ MacCormack } & \multicolumn{2}{c}{ ENO-2 } & \multicolumn{2}{c}{ WENO-5 } \\
\cline { 2 - 8 } Grid & Area & \% loss & Area & \% loss & Area & \% loss & Area & \% loss \\
\hline $40^{2}$ & $2 \cdot 36-02$ & $40 \cdot 93$ & $4 \cdot 01-02$ & $-0 \cdot 27$ & $3 \cdot 36-02$ & 15.96 & $3 \cdot 66-02$ & $8 \cdot 58$ \\
$80^{2}$ & $3 \cdot 06-02$ & $23 \cdot 39$ & $0 \cdot 04-02$ & $-0 \cdot 19$ & $3 \cdot 70-02$ & $7 \cdot 48$ & $3 \cdot 87-02$ & $3 \cdot 13$ \\
$160^{2}$ & $3 \cdot 48-02$ & $12 \cdot 97$ & $0 \cdot 04-02$ & $-0 \cdot 034$ & $3 \cdot 87-02$ & $3 \cdot 21$ & $3 \cdot 96-02$ & $1 \cdot 06$ \\
$320^{2}$ & $3 \cdot 72-02$ & $7 \cdot 00$ & $0 \cdot 04-02$ & $0 \cdot 016$ & $3 \cdot 95-02$ & $1 \cdot 34$ & $3 \cdot 99-02$ & $0 \cdot 35$ \\
\hline
\end{tabular}




$$
\begin{array}{r}
\left.\begin{array}{r}
u(x, y, t)=\sin (x) \cos (y) \\
v(x, y, t)=-\cos (x) \sin (y)
\end{array}\right\} \text { for } 0 \leq t \leq T / 2, \\
\left.\begin{array}{r}
u(x, y, t)=-\sin (x) \cos (y) \\
v(x, y, t)=\cos (x) \sin (y)
\end{array}\right\} \text { for } T / 2<t \leq T .
\end{array}
$$

The above velocity field constitutes a time reversed shear flow field defined in the domain $\Omega=[0, \pi] \times[0, \pi]$. A circular interface of radius $0 \cdot 2 \pi$ is placed in this velocity field with its center initially positioned at $(0 \cdot 5 \pi, 0 \cdot 2(\pi+1))$. The interface is then advected forward so that it undergoes a large distortion for a certain time, say $T / 2$, after which the velocities are reversed for the same length of time in order to return the interface to its initial undistorted position. The time period of the entire operation is $T$. A perfect advection scheme would return the initial interface configuration for time $T$. We perform the test for two time periods, $T=8$ and $T=24$. The $C F L$ number used for the numerical computation is $0 \cdot 2$. In figure 4 we display the results for time period $T=8$ along with prescribed velocity field. Each of the plots in this figure show the initial shape of the interface, the shapes at half and full time period. It is seen that the computed shape and the exact shape are almost indistinguishable for all the case except first-order upwind scheme. For this case the interface undergo only moderate distortion from the initial circular shape before coming back to its initial condition. A second test is performed for the time period $T=24$ during which the interface undergoes large distortion to form a thin filament near the tail before coming back to its initial condition.
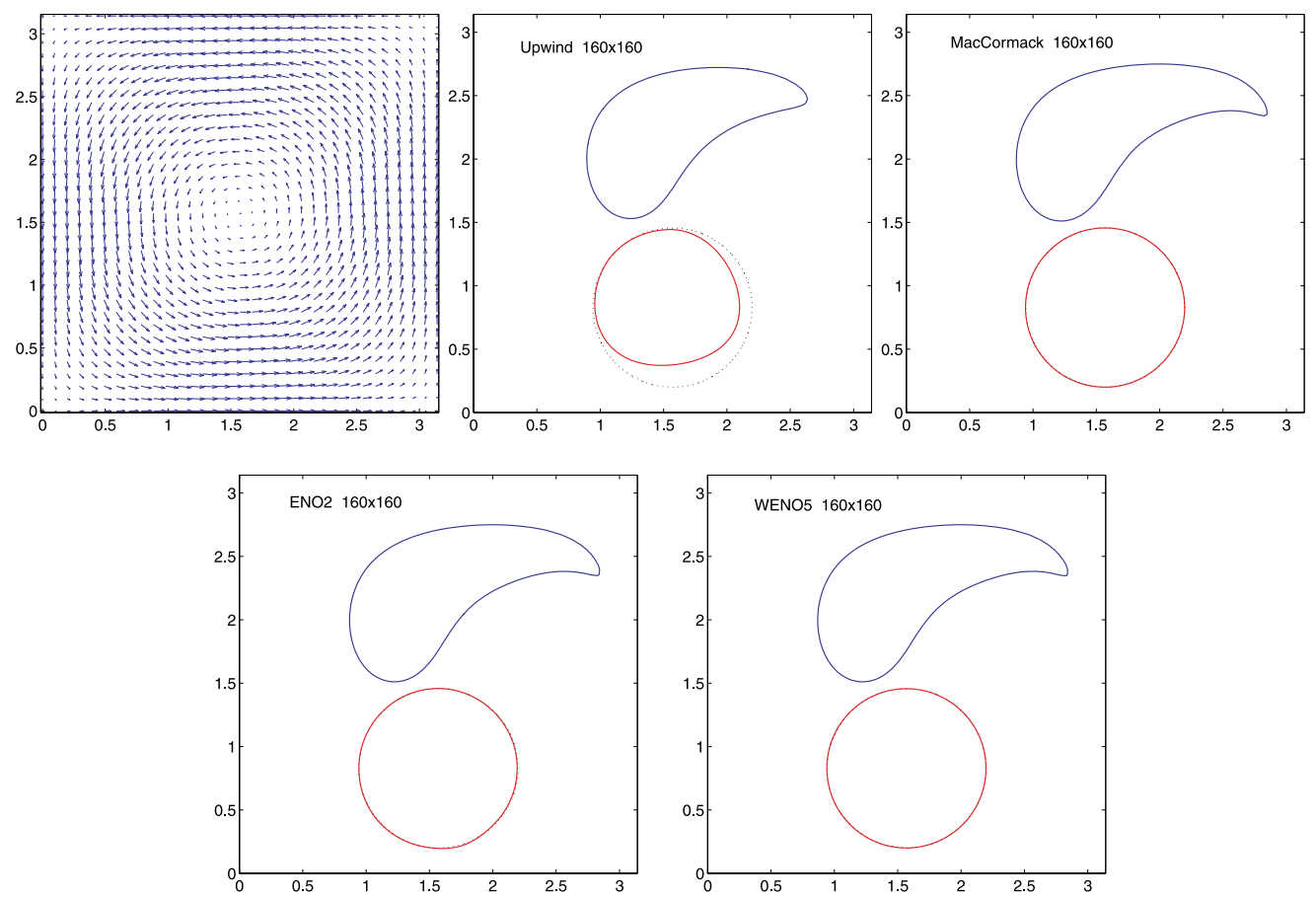

Figure 4. The prescribed time-reversed, shear flow velocity field (top-left); remaining figures display the computed results on $160 \times 160$ grid by various schemes at $t=T / 2$ and $t=T$, for $T=8$. 

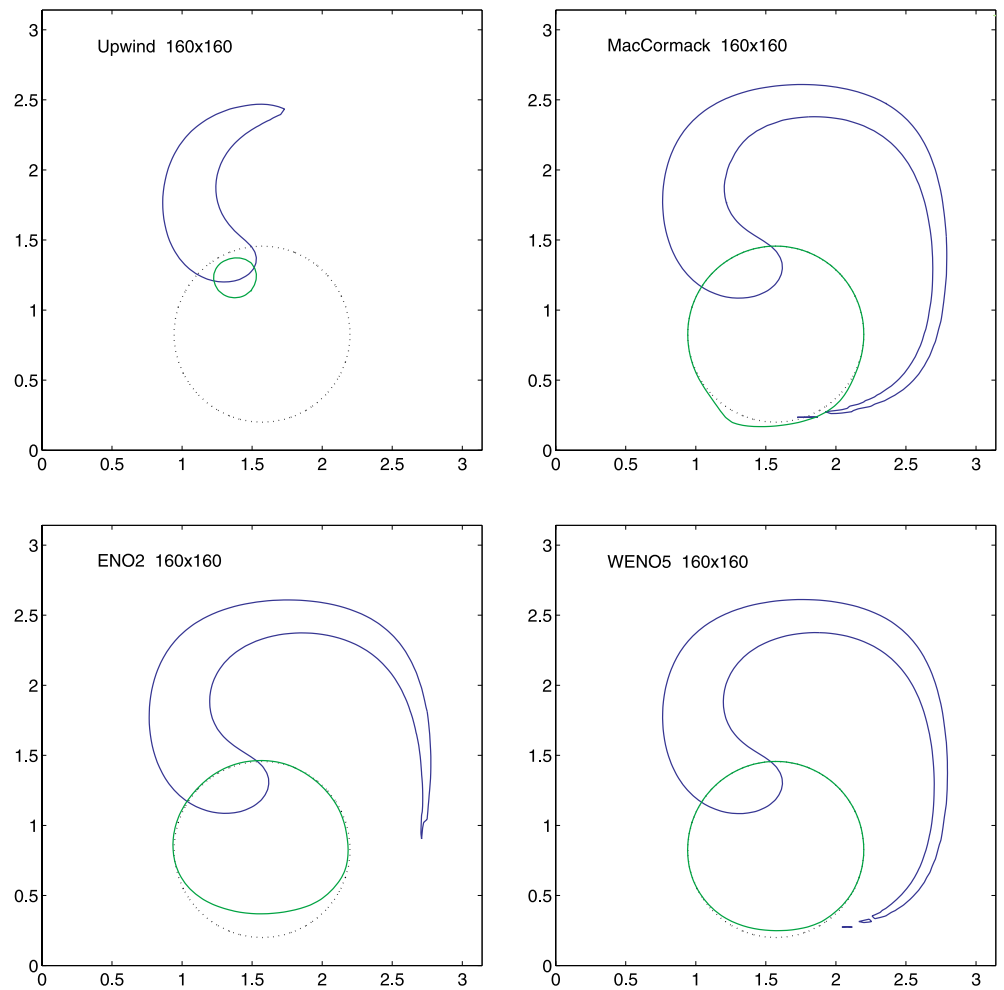

Figure 5. Time-reversed shear field: Computed results on $160 \times 160$ grid by various schemes at $t=T / 2$ and $t=T$, for $T=24$.

The results are shown in figure 5. With upwind scheme, the original circle has reduced to a tiny circle having suffered an area loss of about $94 \%$ on a $160 \times 160$ grid. On coarser grids the circle has simply vanished! ENO-2 scheme also suffers from high $\%$ of area loss. With MacCormack's and WENO-5 schemes the resulting interface at the end of computation is close in appearance to the original circle. In terms of area loss the MacCormack's method is slightly superior to WENO-5 scheme. The summary of error in the solution and \% area loss are shown in tables 7 and 8 respectively for both the test cases.

A time history of $\%$ area loss for WENO-5 scheme with Euler time stepping and secondorder Runge-Kutta time stepping on a $160 \times 160$ grid is also shown in figure 6 for comparison. The interface in this case is advected forward until $t=16$. without performing any timereversal. It can be seen that the area conserving property of RK-2 scheme is far superior to that of Euler scheme. In all our future computations we use WENO-5 scheme in conjunction with RK-2 time stepping. We remark here that combining upwind or ENO-2 schemes with RK-2 scheme does not significantly improve the mass conservation property. In fact when upwind scheme is combined with RK-2, the results were found to get worsened.

\subsection{Circular interface in single-vortex field}

In this section we consider a problem which enables us to assess the integrity of the Eulerian interface capturing method more precisely. The problem consists of advecting the interface in a steady, non-uniform vorticity field introduced by Bell et al (1989). This velocity field 
Table 7. Summary of error in $\phi$ for a circular interface placed in the time-reversed, shear flow field using different schemes.

\begin{tabular}{|c|c|c|c|c|c|c|c|c|}
\hline \multirow[b]{2}{*}{ Grid } & \multicolumn{2}{|c|}{ Upwind } & \multicolumn{2}{|c|}{ MacCormack } & \multicolumn{2}{|c|}{ ENO-2 } & \multicolumn{2}{|c|}{ WENO-5 } \\
\hline & $E_{\phi}$ & order & $E_{\phi}$ & order & $E_{\phi}$ & order & $E_{\phi}$ & order \\
\hline & \multicolumn{8}{|c|}{ Time, $t=T$ for $T=8$} \\
\hline $40^{2}$ & 0.887 & - & $2 \cdot 065-02$ & - & $0 \cdot 101$ & - & $4 \cdot 146-02$ & - \\
\hline $80^{2}$ & 0.441 & 1.008 & $2 \cdot 594-03$ & 2.992 & $2 \cdot 578-02$ & 1.970 & $9 \cdot 289-03$ & $2 \cdot 158$ \\
\hline $160^{2}$ & 0.213 & 1.050 & $4.986-04$ & 2.379 & $9 \cdot 964-03$ & $1 \cdot 371$ & $2 \cdot 941-03$ & 1.659 \\
\hline \multirow[t]{2}{*}{$320^{2}$} & $0 \cdot 106$ & 1.007 & $7 \cdot 243-05$ & 2.783 & $4 \cdot 715-03$ & 1.079 & $7 \cdot 245-04$ & $2 \cdot 021$ \\
\hline & \multicolumn{8}{|c|}{ Time, $t=T$ for $T=24$} \\
\hline $40^{2}$ & - & - & $0 \cdot 112$ & - & $0 \cdot 619$ & - & 0.231 & - \\
\hline $80^{2}$ & - & - & $5 \cdot 957-02$ & 0.911 & 0.290 & 1.093 & $0 \cdot 108$ & 1.097 \\
\hline $160^{2}$ & 0.929 & - & $3 \cdot 674-02$ & 0.697 & $0 \cdot 139$ & $1 \cdot 061$ & $2 \cdot 637-02$ & 2.034 \\
\hline $320^{2}$ & $0 \cdot 612$ & 0.602 & $2 \cdot 793-02$ & $0 \cdot 396$ & $6 \cdot 775-02$ & 1.037 & $2 \cdot 952-03$ & $3 \cdot 159$ \\
\hline
\end{tabular}

possesses strong vortical flows that stretch and potentially tear any interface carried within the flow. The vorticity velocity field is centered in the domain $\Omega=[0,1] \times[0,1]$ with the largest velocity located half way to the walls of the domain. Such a velocity field (see top-left plot of figure 7) is given by the equation

$$
u(x, y)=-\sin ^{2}(\pi x) \sin (2 \pi y), \quad v(x, y)=\sin ^{2}(\pi y) \sin (2 \pi x) .
$$

A circular interface of radius 0.15 is placed in this velocity field with its center initially positioned at $(0.5,0.75)$. The resulting vortex field would spin the fluid elements, stretching

Table 8. Summary of $\%$ area loss for a circular interface placed in the time-reversed, shear flow field. Exact area enclosing the interface $=0.12566$.

\begin{tabular}{|c|c|c|c|c|c|c|c|c|}
\hline \multirow[b]{2}{*}{ Grid } & \multicolumn{2}{|c|}{ Upwind } & \multicolumn{2}{|c|}{ MacCormack } & \multicolumn{2}{|c|}{ ENO-2 } & \multicolumn{2}{|c|}{ WENO-5 } \\
\hline & Area & $\%$ loss & Area & $\%$ loss & Area & $\%$ loss & Area & $\%$ loss \\
\hline & \multicolumn{8}{|c|}{ Time, $t=T$ for $T=8$} \\
\hline $40^{2}$ & 0.0131 & 89.55 & $0 \cdot 1257$ & $-0 \cdot 056$ & $0 \cdot 1144$ & 8.99 & 0.1223 & $2 \cdot 65$ \\
\hline $80^{2}$ & 0.0695 & 44.71 & $0 \cdot 1257$ & -0.007 & $0 \cdot 1229$ & $2 \cdot 21$ & 0.1255 & $0 \cdot 122$ \\
\hline $160^{2}$ & 0.0985 & 21.56 & $0 \cdot 1257$ & -0.0009 & $0 \cdot 1255$ & $0 \cdot 148$ & $0 \cdot 1256$ & 0.003 \\
\hline \multirow[t]{2}{*}{$320^{2}$} & $0 \cdot 1123$ & $10 \cdot 63$ & $0 \cdot 1257$ & -0.0001 & $0 \cdot 1256$ & 0.035 & $0 \cdot 1256$ & 0.0003 \\
\hline & \multicolumn{8}{|c|}{ Time, $t=T$ for $T=24$} \\
\hline $40^{2}$ & 0.0 & $100 \cdot 0$ & $0 \cdot 1224$ & 2.59 & 0.0535 & 57.44 & $0 \cdot 1009$ & 19.73 \\
\hline $80^{2}$ & 0.0 & $100 \cdot 0$ & 0.1244 & 1.01 & 0.0946 & 24.74 & 0.1106 & 11.99 \\
\hline $160^{2}$ & 0.0071 & $94 \cdot 35$ & $0 \cdot 1255$ & $0 \cdot 124$ & $0 \cdot 1112$ & 11.54 & $0 \cdot 1223$ & 2.68 \\
\hline $320^{2}$ & 0.0525 & 58.21 & $0 \cdot 1250$ & 0.505 & $0 \cdot 1194$ & $5 \cdot 01$ & 0.1254 & $0 \cdot 184$ \\
\hline
\end{tabular}




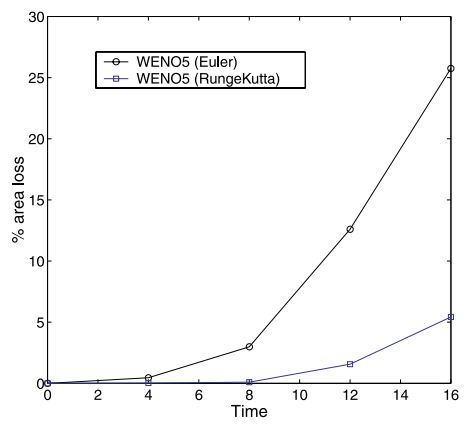

Figure 6. Comparison of area loss for WENO-5 scheme with Euler time stepping and Runge-Kutta time stepping on $160 \times$ 160 grid, without time reversal.

the circular interface into a very long filament that spirals about the center of the domain. The $C F L$ number used for the numerical computation is $0 \cdot 32$. Figures 7 and 8 display the shape of the interface at time $t=1$ and $t=3$ respectively along with the initial shape. Even during the relatively short time interval of $t=1$, there has been a substantial amount of stretching of interface. By a time interval of $t=3$, the spiral wraps around approximately two and a half times (see figure 8). Though a quantitative assessment of the errors in $\phi$ is not possible, we can study the area conservation properties of the interface capturing schemes. This test seems to be a very stringent one as far as the ability of the scheme to maintain thin, elongated
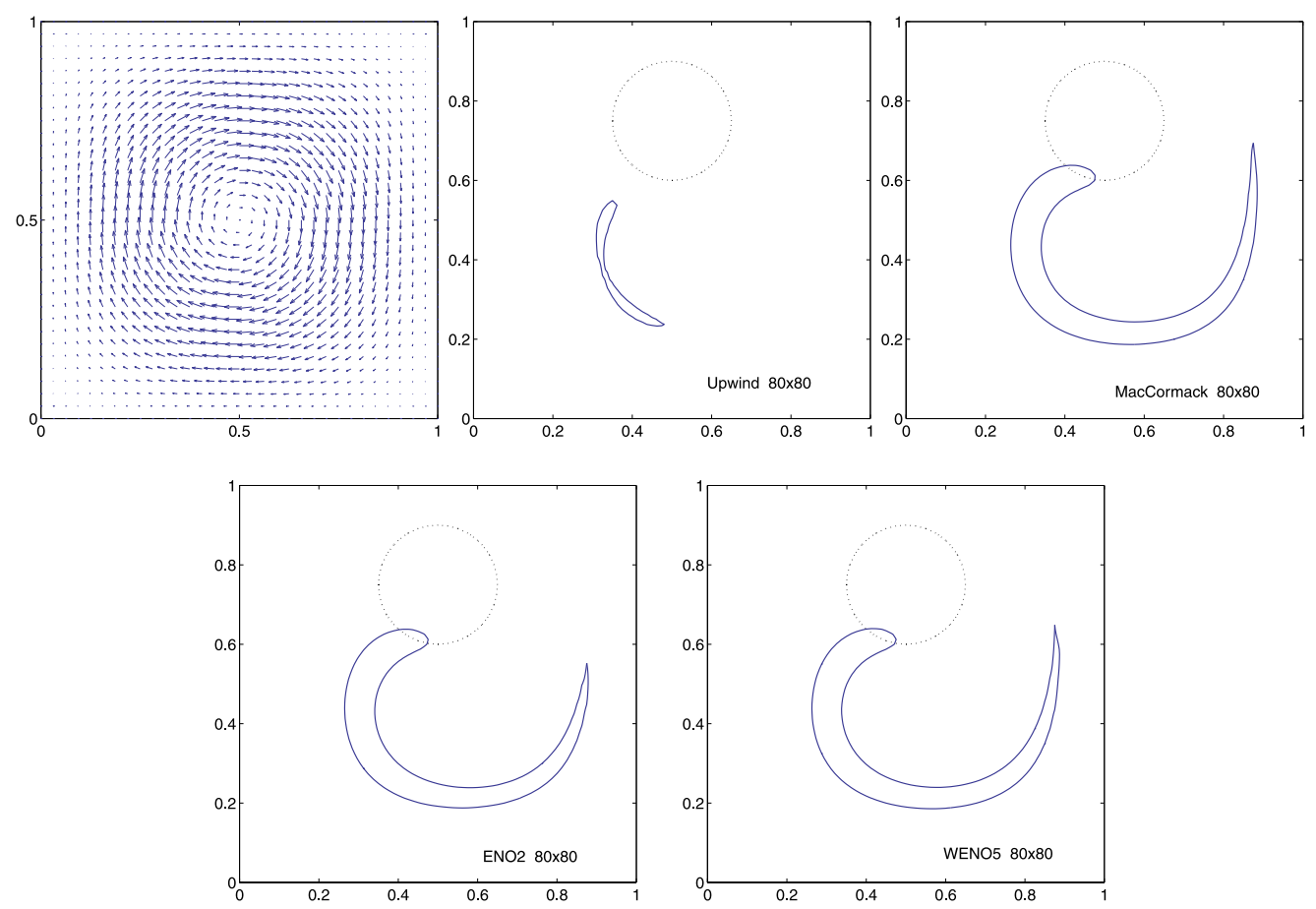

Figure 7. The prescribed single-vortex flow field (top-left); remaining figures display the computed results on $80 \times 80$ grid by various schemes at $t=1$. 

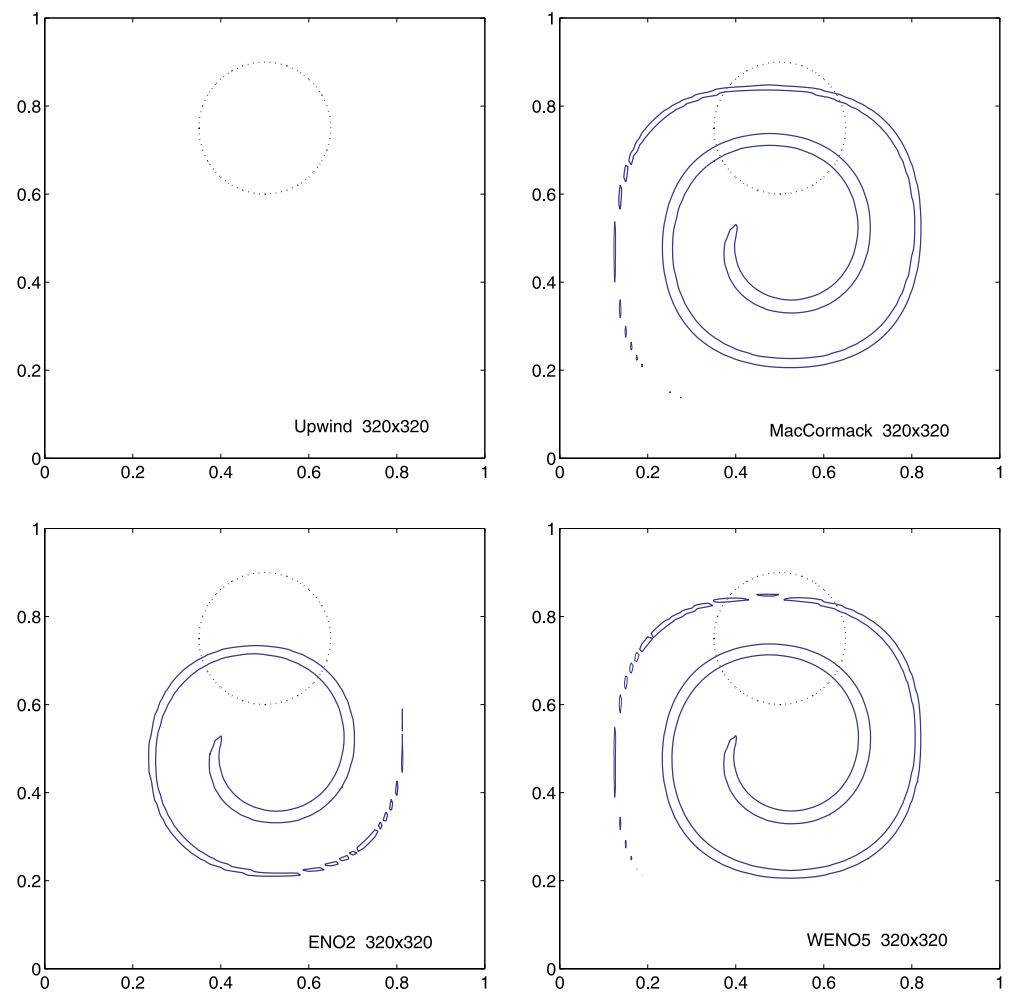

Figure 8. Single-vortex field: Computed results on $320 \times 320$ grid by various schemes at $t=3$.

filaments for longer times. As can be seen from table 9 for $t=1$, the results are good for all schemes except the lower order upwind scheme. However, for $t=3$ the use of upwind scheme completely wipes out the interface even on a relatively fine mesh of $320 \times 320$. The second-order ENO scheme is also severely affected. It conserves only about $60 \%$ of the original area on $320 \times 320$ mesh. Again, MacCormack's scheme exhibits excellent area preserving property. WENO-5 scheme's area preserving property markedly improves with mesh refinement and is able to conserve about $97 \%$ of the original area on $320 \times 320$ mesh. The shape of the interface computed by MacCormack's and WENO-5 schemes at $t=3$ agrees well with that of Rider \& Kothe (1998) using VOF PLIC method and Enright et al (2002) using particle level set method.

\subsection{Circular interface in time-reversed single-vortex field}

Following LeVeque (1996), the single-vortex flow field considered in the last section can be modified by multiplying by the term $\cos (\pi t / T)$. This causes the flow to return to its initial state at time $t=T$ and thus enable us to make a quantitative comparison of errors in the computed solution of $\phi$. The maximum distortion from initial shape occurs at $t=T / 2$. As the reversal period $T$ becomes longer, the interface stretches further away from its initial circular shape. It may be noted that, because of the presence of periodic term $\cos (\pi t / T)$, the resulting vortical field imparts less distortion to the interface as compared to the steady vortical field 
Table 9. Summary of \% area loss for a circular interface placed in the single-vortex field. Exact area enclosing the interface $=0.070686$.

\begin{tabular}{|c|c|c|c|c|c|c|c|c|}
\hline \multirow[b]{2}{*}{ Grid } & \multicolumn{2}{|c|}{ Upwind } & \multicolumn{2}{|c|}{ MacCormack } & \multicolumn{2}{|c|}{ ENO-2 } & \multicolumn{2}{|c|}{ WENO-5 } \\
\hline & Area & $\%$ loss & Area & $\%$ loss & Area & $\%$ loss & Area & $\%$ loss \\
\hline & \multicolumn{8}{|c|}{ Time, $t=1$} \\
\hline $40^{2}$ & 0.0 & $100 \cdot 0$ & 0.0815 & $-15 \cdot 31$ & 0.0469 & 33.59 & 0.0651 & 7.85 \\
\hline $80^{2}$ & 0.0091 & 87.05 & 0.0724 & -2.51 & 0.0652 & 7.78 & 0.0697 & $1 \cdot 33$ \\
\hline $160^{2}$ & 0.0435 & 38.47 & 0.0702 & 0.656 & 0.0698 & $1 \cdot 19$ & 0.0706 & 0.056 \\
\hline \multirow[t]{2}{*}{$320^{2}$} & 0.0601 & 14.90 & 0.0706 & 0.015 & 0.0706 & 0.097 & 0.0706 & 0.034 \\
\hline & \multicolumn{8}{|c|}{ Time, $t=3$} \\
\hline $40^{2}$ & $0 \cdot 0$ & $100 \cdot 0$ & 0.0682 & 3.55 & 0.0 & $100 \cdot 0$ & 0.0021 & 97.09 \\
\hline $80^{2}$ & 0.0 & $100 \cdot 0$ & 0.0752 & -6.41 & 0.0 & 99.97 & 0.0211 & $70 \cdot 18$ \\
\hline $160^{2}$ & $0 \cdot 0$ & $100 \cdot 0$ & 0.0782 & $-10 \cdot 72$ & 0.0059 & 91.53 & 0.0518 & $26 \cdot 71$ \\
\hline $320^{2}$ & $0 \cdot 0$ & $100 \cdot 0$ & 0.0737 & $-4 \cdot 35$ & 0.0425 & $39 \cdot 88$ & 0.0684 & $3 \cdot 28$ \\
\hline
\end{tabular}

for the same time duration $T / 2$. The test details are the same as that of single-vortex test described previously. The modified time-reversed velocity field is given by

$$
\begin{aligned}
& u(x, y, t)=-\sin ^{2}(\pi x) \sin (2 \pi y) \cos (\pi t / T), \\
& v(x, y, t)=\sin ^{2}(\pi y) \sin (2 \pi x) \cos (\pi t / T) .
\end{aligned}
$$

We perform the test for two time periods, $T=2$ and $T=8$. The $C F L$ number used for the numerical computation is $0 \cdot 32$. In figure 9 we display the results for time period $T=2$ performed on a $80 \times 80$ mesh, along with prescribed velocity field and the shape of the interface at $t=T / 2$. It is again seen that the computed shape and the exact shape are almost indistinguishable for all the cases except first-order upwind scheme. The error in the solution of $\phi$ and the loss of area are summarized in tables 10 and 11 respectively.

Figure 10 display the computed results for $T=8$ on a $320 \times 320$ mesh. The top-left plot in this figure shows the shape of the interface corresponding to the maximum stretching (at $t=4$ ) before the time is reversed. It can be seen that the initial circular interface has been transformed to a long thin filament of fluid spiraling inward towards the vortex center. As in the case of steady vortex field, the upwind scheme produces too much numerical diffusion and completely swallows the interface. The ENO-2 scheme too performs so badly as about $40 \%$ of the area is lost even on a relatively fine grid of $320 \times 320$. However, the MacCormack and WENO-5 schemes hold up together at least on finer grid. This demonstrates that under the extreme circumstances, the level set advection equation has to be solved by either nondiffusive methods like MacCormack or a very (formally) high order schemes like WENO-5.

\subsection{Circular interface in time-reversed deformation field}

The final test case we consider is even more stringent than the single-vortex test. The problem consists of entrainment of a circular fluid interface in a deformation field containing 16 vortices. The periodic velocity field for this complex flow pattern defined in the domain 

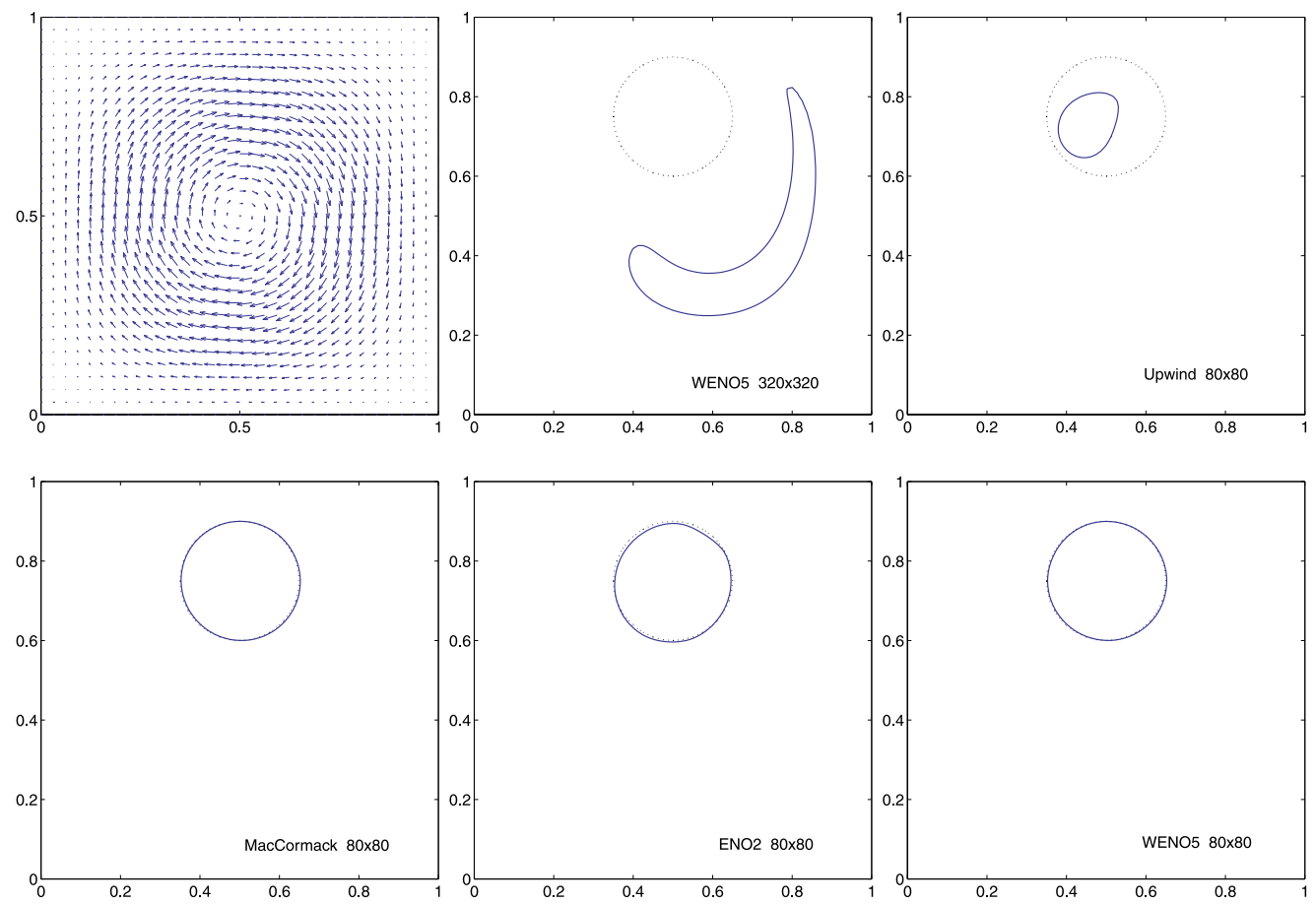

Figure 9. The prescribed time-reversed, single-vortex flow field at $\mathrm{t}=0$ (top-left); the interface shape at $t=0, T / 2$ for $T=2$ (top-center); remaining figures display the computed results on $80 \times 80$ grid by various schemes at $t=T$ for $T=2$.

Table 10. Summary of error in $\phi$ for a circular interface placed in the time-reversed, single-vortex field using different schemes.

\begin{tabular}{|c|c|c|c|c|c|c|c|c|}
\hline \multirow[b]{2}{*}{ Grid } & \multicolumn{2}{|c|}{ Upwind } & \multicolumn{2}{|c|}{ MacCormack } & \multicolumn{2}{|c|}{ ENO-2 } & \multicolumn{2}{|c|}{ WENO-5 } \\
\hline & $E_{\phi}$ & order & $E_{\phi}$ & order & $E_{\phi}$ & order & $E_{\phi}$ & order \\
\hline & \multicolumn{8}{|c|}{ Time, $t=T$ for $T=2$} \\
\hline $40^{2}$ & $7 \cdot 559-02$ & - & $3 \cdot 251-03$ & - & $1 \cdot 512-02$ & - & $4.697-03$ & - \\
\hline $80^{2}$ & $5 \cdot 507-02$ & 0.457 & $1 \cdot 687-03$ & 0.946 & $4 \cdot 468-03$ & 1.759 & $1.740-03$ & 1.433 \\
\hline $160^{2}$ & $2 \cdot 482-02$ & $1 \cdot 150$ & $8 \cdot 498-04$ & 0.989 & $1 \cdot 316-03$ & 1.763 & $8 \cdot 553-04$ & 1.025 \\
\hline \multirow[t]{2}{*}{$320^{2}$} & $1 \cdot 203-02$ & 1.045 & $4 \cdot 257-04$ & 0.997 & $6 \cdot 409-04$ & 1.038 & $4 \cdot 061-04$ & 1.074 \\
\hline & \multicolumn{8}{|c|}{ Time, $t=T$ for $T=8$} \\
\hline $40^{2}$ & - & - & $1.969-02$ & - & - & - & $7 \cdot 561-02$ & - \\
\hline $80^{2}$ & - & - & $7 \cdot 215-03$ & 1.448 & - & - & $3 \cdot 770-02$ & 1.004 \\
\hline $160^{2}$ & - & - & $2 \cdot 759-03$ & 2.615 & $6 \cdot 875-02$ & - & $1 \cdot 381-02$ & 1.448 \\
\hline $320^{2}$ & - & - & $5 \cdot 923-04$ & 2.219 & $3 \cdot 096-02$ & $1 \cdot 151$ & $1 \cdot 825-03$ & 2.919 \\
\hline
\end{tabular}


Table 11. Summary of $\%$ area loss for a circular interface placed in the time-reversed, single-vortex field. Exact area enclosing the interface $=0.070686$.

\begin{tabular}{|c|c|c|c|c|c|c|c|c|}
\hline \multirow[b]{2}{*}{ Grid } & \multicolumn{2}{|c|}{ Upwind } & \multicolumn{2}{|c|}{ MacCormack } & \multicolumn{2}{|c|}{ ENO-2 } & \multicolumn{2}{|c|}{ WENO-5 } \\
\hline & Area & $\%$ loss & Area & $\%$ loss & Area & $\%$ loss & Area & $\%$ loss \\
\hline & \multicolumn{8}{|c|}{ Time, $t=T$ for $T=2$} \\
\hline $40^{2}$ & $0 \cdot 0$ & $100 \cdot 0$ & 0.0711 & -0.544 & 0.0585 & $17 \cdot 24$ & 0.0674 & $4 \cdot 62$ \\
\hline $80^{2}$ & 0.0189 & $73 \cdot 28$ & 0.0707 & -0.037 & 0.0685 & 3.09 & 0.0705 & 0.211 \\
\hline $160^{2}$ & $0 \cdot 0484$ & 31.48 & 0.0707 & -0.004 & 0.0706 & $0 \cdot 111$ & 0.0707 & 0.008 \\
\hline \multirow[t]{2}{*}{$320^{2}$} & $0 \cdot 0611$ & $13 \cdot 59$ & 0.0707 & -0.0005 & 0.0706 & 0.021 & 0.0707 & $0 \cdot 0003$ \\
\hline & \multicolumn{8}{|c|}{ Time, $t=T$ for $T=8$} \\
\hline $40^{2}$ & $0 \cdot 0$ & $100 \cdot 0$ & 0.0562 & $20 \cdot 52$ & 0.0 & $100 \cdot 0$ & $0 \cdot 0$ & $100 \cdot 0$ \\
\hline $80^{2}$ & $0 \cdot 0$ & $100 \cdot 0$ & 0.0716 & $-1 \cdot 31$ & 0.0 & $100 \cdot 0$ & 0.0258 & 63.48 \\
\hline $160^{2}$ & $0 \cdot 0$ & $100 \cdot 0$ & 0.0722 & $-2 \cdot 18$ & 0.0057 & 91.85 & 0.0540 & $23 \cdot 56$ \\
\hline $320^{2}$ & 0.0 & $100 \cdot 0$ & 0.0708 & -0.237 & 0.0421 & 40.42 & 0.0691 & $2 \cdot 23$ \\
\hline
\end{tabular}
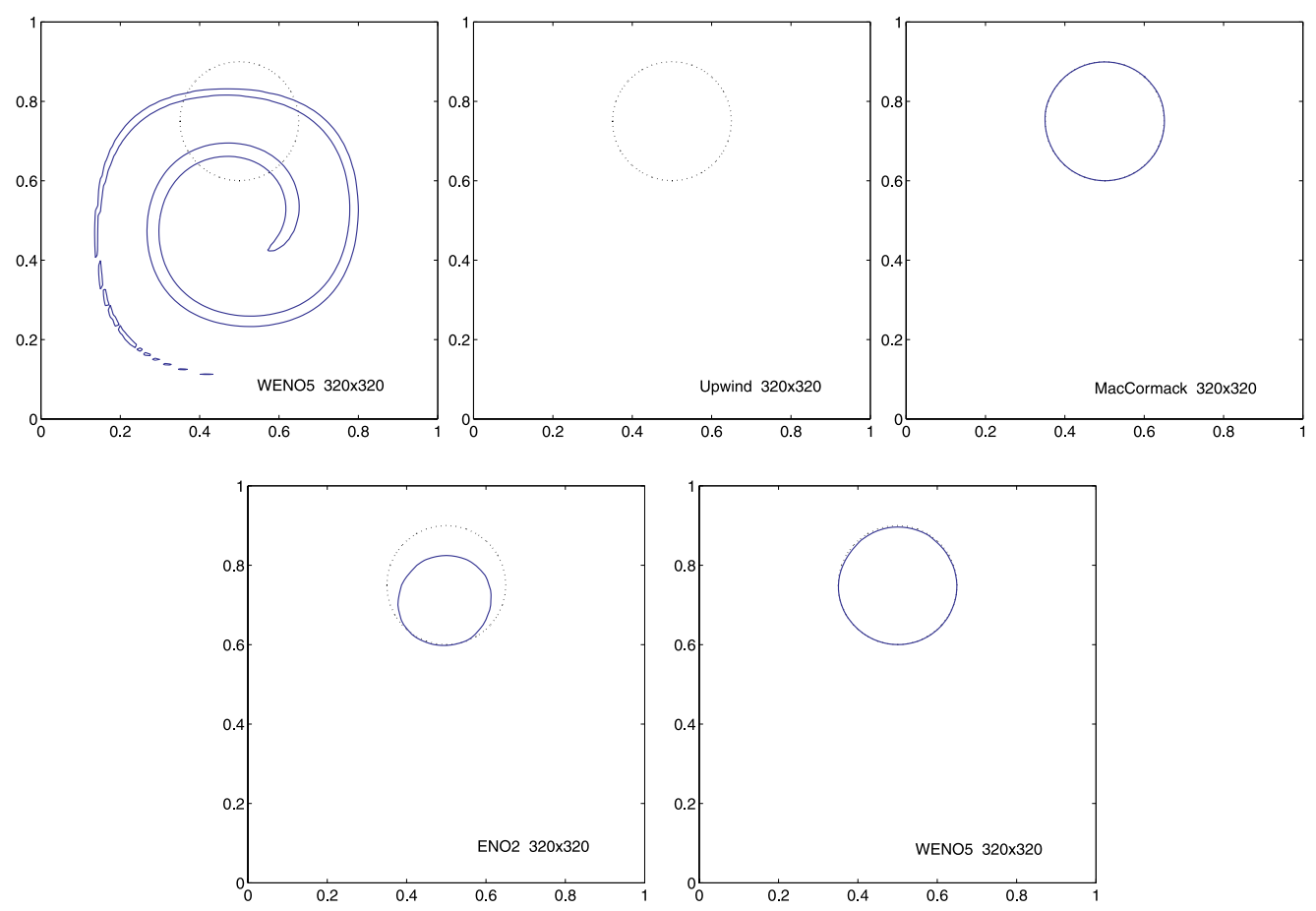

Figure 10. Time-reversed, single-vortex flow field: The interface shape at $t=0, T / 2$ for $T=8$ (top-center); remaining figures display the computed results on $320 \times 320$ grid by various schemes at $t=T$ for $T=8$. 
$\Omega=[0,1] \times[0,1]$ was proposed by Smolarkiewicz (1982). The time-reversed version of it is given by

$$
\begin{aligned}
& u(x, y, t)=\sin (4 \pi(x+1 / 2)) \sin (4 \pi(y+1 / 2)) \cos (\pi t / T), \\
& v(x, y, t)=\cos (4 \pi(x+1 / 2)) \cos (4 \pi(y+1 / 2)) \cos (\pi t / T)
\end{aligned}
$$

and is shown in figure 11 . This velocity field has the ability to force the circular interface of radius 0.25 positioned at $(0.5,0.75)$ to undergo extreme topological changes by a period of time $t=3$. By this time, a large fraction of the interface has been entrained into the two nearest vortices, with a smaller portion, consisting of thin filaments entrapped by two nearby vortices. Since the periodicity is enforced the interface crosses the top boundary of domain and reappear on the bottom (see figure 11). Computations were made on various meshes with $C F L$ number 0.25 for $T=2$. The results for $320 \times 320$ mesh at $t=1$ are displayed in figure 11. The results after reversing time are displayed in figure 12. Note that the width of some of the fluid filaments are of the order of a grid cell and would be very difficult to resolve on an Eulerian grid.

However, it is encouraging to see that the results of different schemes are qualitatively the same for $t=1$ though some finer filaments are not visible in upwind and ENO-2 results. It is surprising to note that the MacCormack solution at $t=2$ falls right on the initial circle thus outperforming the WENO-5 scheme. We found that the convergence to the solution is
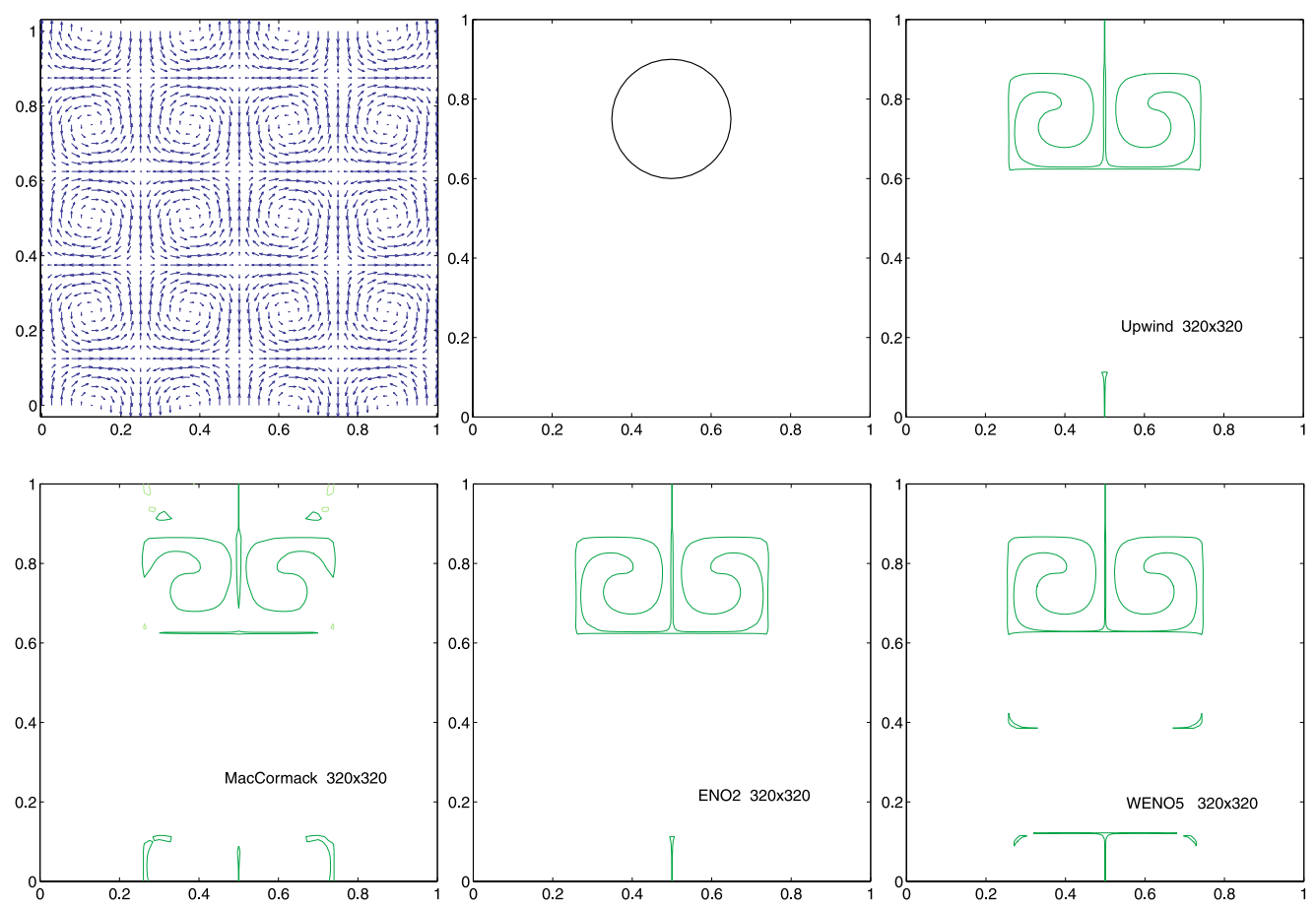

Figure 11. The prescribed periodic, deformation flow field at $t=0$ (top-left); the interface shape at $t=0$ (top-center); remaining figures display the computed results on $320 \times 320$ grid by various schemes at $t=T / 2$ for $T=2$. 

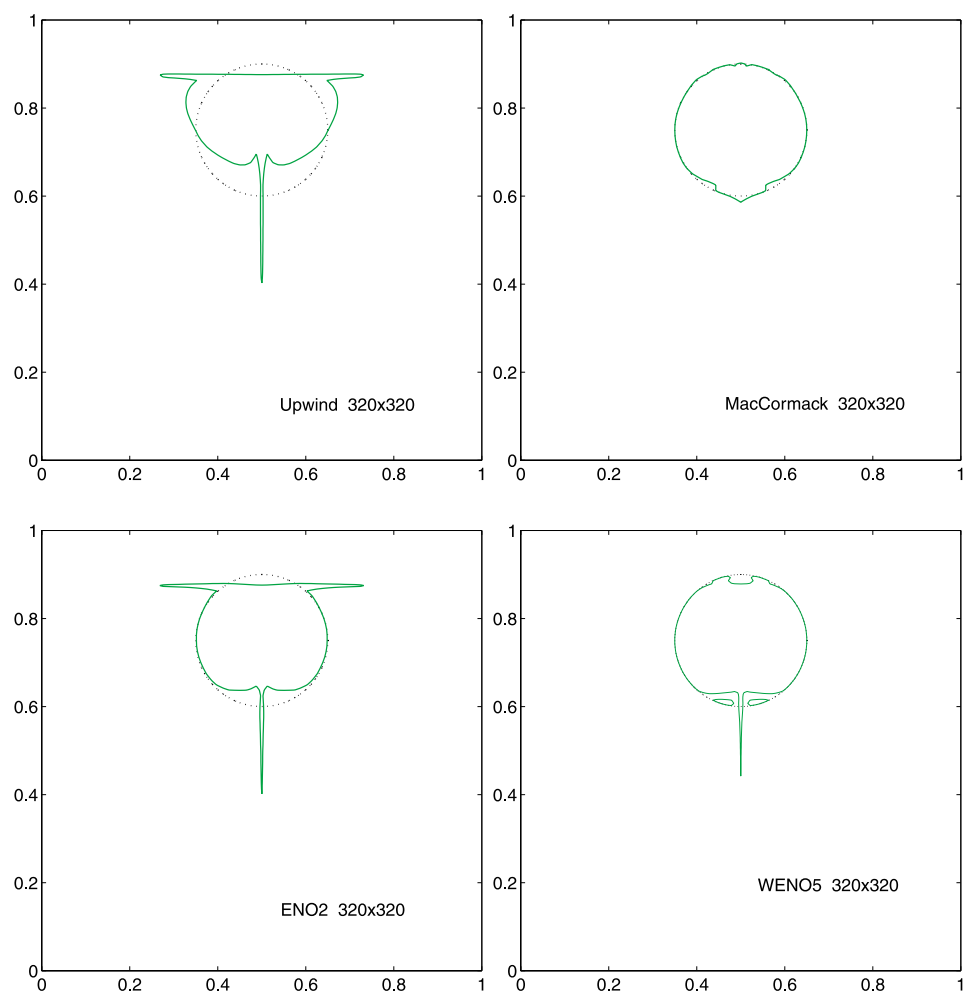

Figure 12. Display of the computed results on $320 \times 320$ grid by various schemes at $t=T$ for $T=2$.

very slow with all the schemes for this difficult test problem. A very fine grid is required to resolve all the minor details of the thin filaments on an Eulerian mesh. The summary of area loss is given in table 12 .

\section{Summary and conclusions}

A thorough testing and assessment of level set based advection of interfaces using different schemes has been performed. The emphasis was given on how the shape and integrity of interfaces is maintained when exposed to complex flow field. Since mass (area enclosing the interface in our tests) conservation is also a major issue in Eulerian type interface capturing methods, we have quantified the percentage of mass loss in all our test cases. We have considered a variety of tests; some simple and other capable of inducing gross interface topology changes. Results of our test indicates that the highly diffusive first-order upwind scheme is totally useless under all circumstances. Many researchers in the past have used ENO-2 scheme for level set advection. However, this scheme also performs badly, especially on coarser grids, when exposed to shearing and vortical velocity fields. Mass conservation property of ENO-2 scheme is also poor unless the grid is very fine. MacCormack's scheme provides excellent results in preserving the interface shapes and with regards to mass conservation when exposed to shearing and vortical flows. However, its performance is not satisfactory when interface 
Table 12. Summary of $\%$ area loss for a circular interface placed in the time-reversed, periodic, deformation field. Exact area enclosing the interface $=0.070686$.

\begin{tabular}{|c|c|c|c|c|c|c|c|c|}
\hline \multirow[b]{2}{*}{ Grid } & \multicolumn{2}{|c|}{ Upwind } & \multicolumn{2}{|c|}{ MacCormack } & \multicolumn{2}{|c|}{ ENO-2 } & \multicolumn{2}{|c|}{ WENO-5 } \\
\hline & Area & $\%$ loss & Area & $\%$ loss & Area & $\%$ loss & Area & $\%$ loss \\
\hline & \multicolumn{8}{|c|}{ Time, $t=T / 2$ for $T=2$} \\
\hline $40^{2}$ & 0.0497 & $29 \cdot 60$ & $0 \cdot 1696$ & $-140 \cdot 43$ & $0 \cdot 0660$ & 6.639 & 0.0693 & 1.988 \\
\hline $80^{2}$ & 0.0594 & $15 \cdot 87$ & 0.1253 & -77.27 & 0.0671 & 4.993 & 0.0693 & 1.921 \\
\hline $160^{2}$ & 0.0640 & 9.458 & $0 \cdot 1029$ & $-45 \cdot 66$ & 0.0664 & $6 \cdot 051$ & 0.0681 & 3.713 \\
\hline \multirow[t]{2}{*}{$320^{2}$} & 0.0647 & 8.405 & 0.0852 & $-20 \cdot 55$ & 0.0662 & $6 \cdot 376$ & 0.0697 & $1 \cdot 354$ \\
\hline & \multicolumn{8}{|c|}{ Time, $t=T$ for $T=2$} \\
\hline $40^{2}$ & 0.0371 & $47 \cdot 52$ & 0.0808 & -14.33 & 0.0547 & $22 \cdot 67$ & 0.0600 & $15 \cdot 07$ \\
\hline $80^{2}$ & 0.0362 & $48 \cdot 74$ & 0.0753 & -6.633 & 0.0632 & $10 \cdot 56$ & 0.0672 & 4.874 \\
\hline $160^{2}$ & 0.0545 & $22 \cdot 89$ & 0.0727 & $-2 \cdot 811$ & $0 \cdot 0642$ & $9 \cdot 217$ & 0.0656 & $7 \cdot 191$ \\
\hline $320^{2}$ & 0.0607 & 14.08 & 0.0715 & $-1 \cdot 155$ & $0 \cdot 0645$ & 8.793 & 0.0674 & 4.643 \\
\hline
\end{tabular}

involving sharp corners are placed in simple advection fields. Figures 2 and 3 demonstrate this. The higher-order WENO-5 scheme coupled with second-order Runge-Kutta time stepping provides the most consistent performance under all situations. Its mass conservation property markedly improves when the mesh is being refined. It is able to advect the sharp interfaces with minimum diffusion. This property is important in simulation of interfacial flows with sharp corners and cusps.

Finally, with regard to the order of convergence of various schemes, a few words are in order. Our numerical experiments demonstrate that the order of convergence of solution $\phi$ is highly problem-dependent. For the simple advection test of circular interface, the upwind scheme shows a first-order convergence as expected. Also, as expected, both MacCormack and ENO-2 schemes show second-order convergence. However, the formally fifth-order accurate WENO-5 scheme also shows only a second order convergence. This is not surprising (as pointed out by Ubbink \& Issaa (1999) in the context of their CICSAM methodology) since the interface is captured over two to three cells at all times irrespective of the mesh density. Thus the error in capturing the interface on an Eulerian mesh can diminish proportionally to the reduction in the cell width. When the interface involves the sharp corners, the order of convergence has slightly reduced with all the schemes (see tables 3 and 5). It can be seen that, for time-reversed shear flow and single-vortex fields, the WENO-5 scheme shows the third-order convergence rate on fine grids.

In summary, the numerical results for these stringent tests provide ample evidence that the level set interface capturing scheme performs excellently when higher-order schemes are used for the solution of advection equation. We also note that for certain type of problems mass conservation is an issue on coarser meshes even with higher order schemes. However, with mesh refinement this problem can be alleviated provided the additional computational effort can be met. Computational cost can be prohibitively expensive on fine grids, when the Level set equation is coupled to the Navier-Stokes equation to simulate multi-phase flows. 


\section{Appendix I}

The key feature of any upwind type scheme is to approximate derivatives by biasing the finite difference stencil in the direction where the characteristics information is coming from. An upwind type scheme for the level set equation 1 on a uniform finite difference grid can be written in the following form

$$
\left(\frac{\delta \phi}{\delta t}\right)_{i, j}+\left(u^{+} \phi_{x}^{-}+u^{-} \phi_{x}^{+}+v^{+} \phi_{y}^{-}+v^{-} \phi_{y}^{+}\right)=0,
$$

where $(\delta \phi / \delta t)$ represents the temporal discretization operator and

$$
\begin{aligned}
u^{+} & =\max \left(u_{i, j}, 0\right), & u^{-}=\min \left(u_{i, j}, 0\right), \\
v^{+} & =\max \left(v_{i, j}, 0\right), & v^{-}=\min \left(v_{i, j}, 0\right) .
\end{aligned}
$$

The definition of $\phi_{x}^{-}, \phi_{x}^{+}$, etc. depends on the type of scheme used to construct the specific finite difference stencil. We give the details for first-order upwind scheme, second-order ENO scheme, and fifth-order WENO scheme.

\section{Upwind scheme}

For this basic first-order scheme, we have

$$
\begin{aligned}
\phi_{x}^{+} & =\left(\frac{\phi_{i+1, j}-\phi_{i, j}}{\Delta x}\right), & \phi_{x}^{-} & =\left(\frac{\phi_{i, j}-\phi_{i-1, j}}{\Delta x}\right), \\
\phi_{y}^{+} & =\left(\frac{\phi_{i, j+1}-\phi_{i, j}}{\Delta y}\right), & \phi_{y}^{-} & =\left(\frac{\phi_{i, j}-\phi_{i, j-1}}{\Delta y}\right) .
\end{aligned}
$$

It is well known that, first-order upwind scheme introduces large amount of diffusive errors.

\section{ENO-2 scheme}

The first order accurate upwind scheme can be improved upon by using more accurate approximation for $\phi_{x}^{-}, \phi_{x}^{+}, \phi_{y}^{-}$, and $\phi_{y}^{+}$. The essentially non-oscillatory or ENO scheme introduced by Harten et al (1987) is one such class of schemes. The key idea of ENO schemes is the use of an adaptive stencil, which is chosen based on the local smoothness of the solution. This adaptive polynomial interpolant is constructed to avoid the use of higher-order polynomial interpolation across a steep gradient in the data. For a second-order ENO scheme on a finite difference grid, $\phi_{x}^{-}$and $\phi_{x}^{+}$are given by

$$
\begin{aligned}
& \phi_{x}^{-}=\frac{\phi_{i, j}-\phi_{i-1, j}}{\Delta x}+\frac{\Delta x}{2} \operatorname{minmod}\left(\frac{\phi_{i+1, j}-2 \phi_{i, j}+\phi_{i-1, j}}{\Delta x^{2}}, \frac{\phi_{i, j}-2 \phi_{i-1, j}+\phi_{i-2, j}}{\Delta x^{2}}\right), \\
& \phi_{x}^{+}=\frac{\phi_{i+1, j}-\phi_{i, j}}{\Delta x}-\frac{\Delta x}{2} \operatorname{minmod}\left(\frac{\phi_{i+1, j}-2 \phi_{i, j}+\phi_{i-1, j}}{\Delta x^{2}}, \frac{\phi_{i+2, j}-2 \phi_{i+1, j}+\phi_{i, j}}{\Delta x^{2}}\right),
\end{aligned}
$$

where the function

$$
\operatorname{minmod}(a, b)= \begin{cases}S(a) \min (|a|,|b|), & \text { if } a \cdot b>0 \\ 0, & \text { otherwise }\end{cases}
$$


and the $S(a)$ is defined as

$$
S(a)= \begin{cases}-1, & \text { if } a<0 \\ 1, & \text { if } a>0 \\ 0, & \text { if } a=0\end{cases}
$$

In an analogous manner we can compute $\phi_{y}^{-}$and $\phi_{y}^{+}$.

\section{WENO-5 scheme}

An improvement of ENO scheme is the weighted ENO (WENO) scheme, which was first developed by Liu et al (1994). Both ENO and WENO use the idea of adaptive stencils in the reconstruction procedure. However, ENO takes just one out of many candidate stencils when doing the reconstruction; while WENO takes a convex combination of all the ENO approximations, each being assigned a non-linear weight which depends on the local smoothness of the numerical solution based on that stencil. Following Osher \& Fedkiw (2003), we give the algorithm for WENO. The third-order accurate ENO scheme uses a subset of $\left\{\phi_{i-3, j}, \phi_{i-2, j}, \phi_{i-1, j}, \phi_{i, j}, \phi_{i+1, j}, \phi_{i+2, j}\right\}$ while calculating $\left(\phi_{x}^{-}\right)$. We define

$$
\begin{aligned}
& v_{1}=\frac{\phi_{i-2, j}-\phi_{i-3, j}}{\Delta x}, \\
& v_{2}=\frac{\phi_{i-1, j}-\phi_{i-2, j}}{\Delta x}, \\
& v_{3}=\frac{\phi_{i, j}-\phi_{i-1, j}}{\Delta x}, \\
& v_{4}=\frac{\phi_{i+1, j}-\phi_{i, j}}{\Delta x}, \\
& v_{5}=\frac{\phi_{i+2, j}-\phi_{i+1, j}}{\Delta x}
\end{aligned}
$$

which allows us to write the three possible third-order ENO stencils

$$
\begin{aligned}
\phi_{x}^{1} & =\frac{v_{1}}{3}-\frac{7 v_{2}}{6}+\frac{11 v_{3}}{6}, \\
\phi_{x}^{2} & =-\frac{v_{2}}{6}+\frac{5 v_{3}}{6}+\frac{v_{4}}{3}, \\
\phi_{x}^{3} & =\frac{v_{3}}{3}+\frac{5 v_{4}}{6}-\frac{v_{5}}{6} .
\end{aligned}
$$

The fifth-order WENO approximation of $\left(\phi_{x}^{-}\right)$is then a convex combination of the above three approximation given by

$$
\phi_{x}^{-}=\omega_{1} \phi_{x}^{1}+\omega_{2} \phi_{x}^{2}+\omega_{3} \phi_{x}^{3}
$$

where the $0 \leq \omega_{k} \leq 1$ are the weights with $\omega_{1}+\omega_{2}+\omega_{3}=1$. The weights will be allotted according to the smoothness of the three stencils and are estimated by the following equations 
for smoothness indicators (Jiang \& Peng 2000)

$$
\begin{aligned}
& S_{1}=\frac{13}{12}\left(v_{1}-2 v_{2}+v_{3}\right)^{2}+\frac{1}{4}\left(v_{1}-4 v_{2}+3 v_{3}\right)^{2}, \\
& S_{2}=\frac{13}{12}\left(v_{2}-2 v_{3}+v_{4}\right)^{2}+\frac{1}{4}\left(v_{2}-v_{4}\right)^{2}, \\
& S_{3}=\frac{13}{12}\left(v_{3}-2 v_{4}+v_{5}\right)^{2}+\frac{1}{4}\left(3 v_{3}-4 v_{4}+v_{5}\right)^{2} .
\end{aligned}
$$

Using these smoothness estimates, we define

$$
\begin{aligned}
& \alpha_{1}=\frac{0 \cdot 1}{\left(S_{1}+\epsilon\right)^{2}}, \\
& \alpha_{2}=\frac{0 \cdot 6}{\left(S_{2}+\epsilon\right)^{2}}, \\
& \alpha_{3}=\frac{0 \cdot 3}{\left(S_{3}+\epsilon\right)^{2}}
\end{aligned}
$$

with

$$
\epsilon=10^{-6} \max \left\{v_{1}^{2}, v_{2}^{2}, v_{3}^{2}, v_{4}^{2}, v_{5}^{2}\right\}+10^{-99},
$$

where the $10^{-99}$ term is set to avoid division by zero in the definition of the $\alpha_{k}$. Now, the near optimal weights are obtained by normalizing $\alpha_{k}$ and is given by

$$
\begin{aligned}
& \omega_{1}=\frac{\alpha_{1}}{\alpha_{1}+\alpha_{2}+\alpha_{3}}, \\
& \omega_{2}=\frac{\alpha_{2}}{\alpha_{1}+\alpha_{2}+\alpha_{3}}, \\
& \omega_{3}=\frac{\alpha_{3}}{\alpha_{1}+\alpha_{2}+\alpha_{3}} .
\end{aligned}
$$

In a similar manner, the function $\left(\phi_{x}^{+}\right)_{i, j}$ is constructed with a subset of $\left\{\phi_{i-2, j}, \phi_{i-1, j}, \phi_{i, j}\right.$, $\left.\phi_{i+1, j}, \phi_{i+2, j}, \phi_{i+3, j}\right\}$ (for more details see Osher \& Fedkiw (2003)). The HJ WENO techniques used to approximate the functions $\phi_{x}^{-}$and $\phi_{x}^{+}$can now be applied independently to approximate the functions $\phi_{y}^{-}$and $\phi_{y}^{+}$in a dimension-by-dimension manner.

\section{MacCormack method}

The well known MacCormack method is a predictor-corrector method, that is second order accurate in both time and space. In the predictor step, a temporary predicted value of $\phi$ at $(n+1)$ level is computed using forward Euler scheme while a simple forward differencing is used for approximating spatial derivatives. The corrector step provides the final value of $\phi$ where backward differencing is used for spatial derivatives. The two steps in the scheme are 
given by

$$
\begin{aligned}
& \frac{\phi_{i, j}^{\overline{n+1}}-\phi_{i, j}^{n}}{\Delta t}+u_{i, j}^{n}\left(\frac{\phi_{i+1, j}^{n}-\phi_{i, j}^{n}}{\Delta x}\right)+v_{i, j}^{n}\left(\frac{\phi_{i, j+1}^{n}-\phi_{i, j}^{n}}{\Delta y}\right)=0, \\
& \frac{\phi_{i, j}^{n+1}-\phi_{i, j}^{n+1 / 2}}{\Delta t / 2}+u_{i, j}^{\overline{n+1}}\left(\frac{\phi_{i, j}^{\overline{n+1}}-\phi_{i-1, j}^{\overline{n+1}}}{\Delta x}\right)+v_{i, j}^{\overline{n+1}}\left(\frac{\phi_{i, j}^{\overline{n+1}}-\phi_{i, j-1}^{\overline{n+1}}}{\Delta y}\right)=0,
\end{aligned}
$$

where the time-averaged value of

$$
\phi_{i, j}^{n+1 / 2}=\frac{\phi_{i, j}^{n}+\phi_{i, j}^{\overline{n+1}}}{2} .
$$

The method is stable if $C F L$ number given by equation 2 is less than 1 .

\section{References}

Armenio V 1997 An improved MAC method (SIMAC) for unsteady High-Reynolds free surface flows. Int. J. Numer. Meth. Fluids 24: 185-214

Ashgriz N, Poo J Y 1991 FLAIR: Flux line-segment model for advection and interface reconstruction. J. Comput. Phys. 93: 449-468

Bell J B, Colella P, Glaz H M 1989 A second-order projection method for incompressible NavierStokes equations. J. Comput. Phys. 85: 257-283

Brackbill J U, Kothe D B, Zemach C 1992 A continuum method for modelling surface tension. J. Comput. Phys. 100: 335-354

Chang Y C, Hou T Y, Merriman B, Osher S 1996 A level set formulation of Eulerian interface capturing methods for incompressible fluid flows. J. Comput. Phys. 124: 449-464

Chern I-L, Glimm J, McBryan O, Plohr B, Yaniv S 1986 Front tracking for gasdynamics. J. Comput. Phys. 62: 83-110

Deborah G A 2004 Quadtree adaptive method for simulating fluid flows with moving interfaces. J. Comput. Phys. 194: 35-56

Enright D, Fedkiw R, Ferziger J, Mitchell I 2002 A hybrid particle level set method for improved interface capturing. J. Comput. Phys. 183: 83-116

Fedkiw R, Aslam T, Merriman B, Osher S 1999 A non-oscillatory Eulerian approach to interfaces in multimaterial flows (the ghost fluid method). J. Comput. Phys. 152: 457-492

Glimm J, McBryan O, Menikoff R, Sharp D H 1987 Front tracking applied to Rayleigh-Taylor instability. SIAM J. Sci. Comput. 7: 230-251

Glimm J, Grove J W, Li X-L, Shyue K-M, Zhang Q, Zeng Y 1998 Three-dimensional front tracking. SIAM J. Sci. Comput. 19: 703-727

Harlow F H, Welch J E 1965 Numerical study of large-amplitude free-surface motions. Phys. Fluids 8: 2182-2189

Harten A, Engquist B, Osher S, Chakravarthy S R 1987 Uniformly high order accurate essentially non-oscillatory schemes III. J. Comput. Phys. 71: 231-303

Hou T Y, Li Z, Osher S, Zhao H 1997 A hybrid method for moving interface problems with application to the Hele-Shaw flow. J. Comput. Phys. 134: 236-252

Jiang G-S, Peng D 2000 Weighted ENO Schemes for Hamilton-Jacobi equations. SIAM J. Sci. Comput. 21: $2126-2143$

Juric D, Tryggvason G 1998 Computations of boiling flows. Int. J. Multi-phase Flow 24: 387-410

Kang I S, Leal L G 1987 Numerical solution of axisymmetric unsteady free-boundary problems at finite Reynolds number. I. Finite difference scheme and its application to the deformation of a bubble in a uniaxial straining flow. Phys. Fluids 30: 1929-1940 
Lafaurie B, Nardone C, Scardovelli R, Zaleski S, Zanetti G 1994 Modelling merging and fragmentation in multi-phase flows with SURFER. J. Comput. Phys. 113: 134-147

LeVeque R J, Li Z 1994 The immersed interface methods for elliptic equations with discontinuous coefficients and singular sources. SIAM J. Numer. Anal. 31: 1019-1044

LeVeque R J 1996 High-resolution conservative algorithms for advection in incompressible flow. SIAM J. Numer. Anal. 33: 627-665

Liu X-D, Osher S, Chan T 1994 Weighted essentially non-oscillatory schemes. J. Comput. Phys. 115: 200-212

Mulder W, Osher S J, Sethian J A 1992 Computing interface motion in compressible gasdynamics. J. Comput. Phys. 100: 209-228

Nichols B D, Hirt C W 1975 Methods for calculating multi-dimensional, transient free surface flows past bodies. In Proc. First Int. Conf. Num. Ship Hydrodynamics Gaithersburg 20-23

Osher S, Sethian J A 1988 Fronts propagating with curvature-dependent speed: Algorithm based on Hamilton-Jacobi formulations. J. Comput. Phys. 79: 12-49

Osher S, Fedkiw R 2001 Level set methods: An overview and some recent results. J. Comput. Phys. 169: 463-502

Osher S, Fedkiw R 2003 Level set methods and dynamic implicit surfaces (New York: Springer-Verlag)

Popinet S, Zaleski S 1999 A front tracking algorithm for the accurate representation of surface tension. Int. J. Numer. Meth. Fluids 30: 775-793

Puckett E G, Almgren A S, Bell J B, Marcus D L, Rider W J 1997 A high-order projection method for tracking fluid interfaces in variable density incompressible flows. J. Comput. Phys. 130: 269-282

Rider W J, Kothe D B 1995 Stretching and tearing interface tracking methods in $12^{\text {th }}$ AIAA CFD Conference AIAA-95-1717 San Diego

Rider W J, Kothe D B 1998 Reconstructing volume tracking. J. Comput. Phys. 141: 112-152

Rudman M 1997 Volume tracking methods for interfacial flow calculations. Int. J. Numer. Meth. Fluids 24: 679-691

Scardovelli R, Zaleski S 1999 Direct numerical simulation of free-surface and interfacial flow. Annu. Rev. Fluid Mech. 31: 567-603

Sethian J A 1999 Level set methods and fast marching methods (Cambridge University Press)

Sethian J A, Smereka P 2003 Level set methods for fluid interfaces. Ann. Rev. Fluid Mech. 35: 341-372

Shu C-W, Osher S 1988 Efficient implementation of essentially non-oscillatory shock capturing schemes. J. Comput. Phys. 77: 439-471

Smolarkiewicz P K 1982 The multi-dimensional Crowley advection scheme. Monthly Weather Review 110: $1968-1983$

Sussman M, Smereka P, Osher S 1994 A level set approach for computing solutions to incompressible two-phase flow. J. Comput. Phys. 114: 146-159

Sussman M, Almgren A S, Bell J B, Colella P, Howell L H, Welcome M L 1999 An adaptive level set approach for incompressible two-phase flows. J. Comput. Phys. 148: 81-124

Sussman M, Puckett E G 2000 A coupled level set and volume of fluid method for computing 3D and axisymmetric incompressible two-phase flows. J. Comput. Phys. 162: 301-337

Sussman M, Fatemi E 1999 An efficient, interface-preserving level set Redistancing algorithm and its application to interfacial incompressible fluid flow. SIAM J. Sci. Comput. 20: 1165-1191

Tomé M F, Castelo A, Murakami J, Cuminato J A, Minghim R, Oliveira M C F, Mangiavacchi N, McKee S 2000 Numerical simulation of axisymmetric free surface flows. J. Comput. Phys. 157: $441-472$

Tryggvason G, Unverdi S O 1990 Computations of three-dimensional Rayleigh-Taylor instability. Phys. Fluids A2: 656-659

Tryggvason G, Bunner B, Ebrat O, Tauber W 1998 Computations of multi-phase flows by a finite difference/front tracking method. I. multi-fluid flows. Lecture Notes, Department of Mechanical Engineering and Applied Mechanics, University of Michigan

Ubbink O, Issa R I 1999 A method for capturing sharp fluid interfaces on arbitrary meshes. J. Comput. Phys. 153: 26-50 
Unverdi S O, Tryggvason G 1992 A front-tracking method for viscous, incompressible, multi-fluid flows. J. Comput. Phys. 100: 25-37

Youngs D L 1982 Time-dependent multi-material flow with large fluid distortion, in: Numerical Methods for Fluid Dynamics, (Eds. K W Morton and M J Baines), 273-285, Academic Press

Zhu J, Sethian J A 1992 Projection method coupled to level set interface techniques. J. Comput. Phys. 102: $128-138$ 